\title{
Wave Excited Motion of a Body Floating on Water Confined between Two Semi-infinite Ice Sheets
}

\author{
K. Ren, G.X. Wu a) and G.A. Thomas \\ Department of Mechanical Engineering, University College London, Torrington Place, London WC1E 7JE, \\ United Kingdom \\ a) Electronic mail: g.wu@ucl.ac.uk
}

\begin{abstract}
The wave excited motion of a body floating on water confined between two semiinfinite ice sheets is investigated. The ice sheet is treated as an elastic thin plate and water is treated as an ideal and incompressible fluid. The linearized velocity potential theory is adopted in the frequency domain and problems are solved by the method of matched eigenfunctions expansion. The fluid domain is divided into sub-regions and in each subregion the velocity potential is expanded into a series of eigenfunctions satisfying the governing equation and the boundary conditions on horizontal planes including the free surface and ice sheets. Matching is conducted at the interfaces of two neighbouring regions to ensure the continuity of the pressure and velocity, and the unknown coefficients in the expressions are obtained as a result. The behaviour of the added mass and damping coefficients of the floating body with the effect of the ice sheets, and the excitation force are analysed. They are found to vary oscillatorily with the wave number, which is different from that for a floating body in the open sea. The motion of the body confined between ice sheets is investigated, in particular its resonant behaviour with extremely large motion found to be possible under certain conditions. Standing waves within the polynya are also observed.
\end{abstract}

Key words: ice sheets, free surface waves, floating body, resonances, matched eigenfunctions expansion.

\section{INTRODUCTION}

As commercial shipping routes through the Arctic region may become viable, ${ }^{1}$ there has been increased interest in wave/ice/floating body interaction problems. One of these examples is an open water channel created by an ice-breaker. In such a case, a ship is in fact navigating in a strip of open water between two large ice sheets. When a wave coming from beneath an ice sheet arrives at the open water channel, highly complex interaction between wave/ice/ship 
can occur. The behaviour of a ship in such a case is expected to be very different from that in the open water without any ice sheets and its performance should be properly assessed.

Extensive research has been undertaken on the interaction between ocean waves and sea ice which can be a semi-infinite sheet, a finite floe, lead, polynya, and many other forms. Squire et $a .^{2}$ and Squire ${ }^{3}$ provided overviews on previous studies on this problem with more recent developments discussed by Squire. ${ }^{4}$ In the adopted model, the ice sheet is usually treated as an elastic thin plate and water is treated as an ideal fluid. A very large ice sheet is usually treated as being semi-infinite, with its edge in contact with open water. Fox \& Squire ${ }^{5}$ considered the two-dimensional problem of reflection and transmission of waves from open water to an ice sheet. They adopted the method of eigenfunction expansion for the velocity potentials below the water surface and ice sheet, and used the conjugate gradient method to impose continuity at the interface and edge condition. The reflection and transmission coefficients for wave direction normal to the ice edge were obtained. Later, this method was extended to consider the oblique incidence problem. ${ }^{6}$ Sahoo et al. $^{7}$ reconsidered these problems through defining an inner product with orthogonality based on the eigenfunction expansions method. They solved the problem for the semi-infinite ice with different edge conditions. The problem was also solved by Balmforth \& Craster, ${ }^{8}$ Tkacheva ${ }^{9-10}$ and Chung \& Fox ${ }^{11}$ using the Wiener-Hopf method, based on the formulation derived by Evans \& Davies ${ }^{12}$ and its modification or extension. For the problem of a finite floe interaction with waves, Meylan \& Squire $^{13}$ derived an approximate solution of the reflection and transmission coefficients from the semi-infinite ice sheet model. Later, they used the Green's function method and solved the diffraction problem for a single finite floe, as well as for a pair of finite floes. ${ }^{14}$ While the above work is for ice sheet without the effect of its draught, this effect was taken into account by Williams \& Squire, ${ }^{15}$ Williams \& Porter, ${ }^{16}$ and Williams \& Squire. ${ }^{17}$ The related non-zero draught problem was also considered through a semi-infinite and finite plate by Hermans ${ }^{18}$ using the method of Green's function.

The polynya problem has also been investigated, where a polynya is an area of open water surrounded by sea ice. Chung \& Linton ${ }^{19}$ considered waves propagating through a waterway between two identical semi-infinite elastic plates and found that the reflection coefficients become zero at an infinite number of discrete frequencies. Williams \& Squire ${ }^{20}$ considered ice sheets with different material properties. While the work above focused on the interaction 
between the ice sheets or elastic plates and ocean waves, Sturova ${ }^{21}$ considered the wave generated by an oscillating submerged cylinder in water under a semi-infinite elastic plate and calculated both the hydrodynamic loads on the cylinder and the deformation of the plate, as well as the free surface shape. Based on this method, Sturova ${ }^{22}$ further studied the radiation and diffraction problem of a submerged cylinder in water with a finite-floe or in a polynya.

Rather than a submerged body, the present work considers a body freely floating on the water surface confined between two semi-infinite ice sheets in the context of the physical problem outlined at the beginning of the Introduction. The prime purpose is to investigate how the hydrodynamic coefficients of the body and the excitation force are different from those in open water. This leads to in-depth analysis for the motion of a floating body confined between ice sheets, in particular its unique resonant behaviour. The ice sheets will be treated as elastic plates and the effects of their draughts will be taken into account. The floating body considered is a rectangular box. This allows us to divide the fluid domain into five subregions and the velocity potential in each region is expanded into eigenfunctions. It ought to point out although eigenfucntions expansion method is a classic one, it still has to be used properly to achieve accurate solution efficiently. In fact, it was shown by Sahoo et al. ${ }^{7}$ that the inner product has to be defined properly to ensure orthogonality. Here we shall use the Green's identity to enforce the continuity on the interface and to impose the boundary conditions, and further obtain the unknown coefficients in the expansion. Extensive results are provided through added mass and damping coefficients, excitation force and wave elevation. Their behaviours with the effects of ice sheets and size of the open water are analysed and implications in physics are discussed. In particular, body motion is calculated and its natural frequencies and the subsequent resonances in both coupled and uncoupled motions are investigated.

\section{MATHEMATICAL FORMULATION AND SOLUTION PROCEDURE}

\section{A. Governing equation and boundary conditions}

We consider the problem of a body floating on the open water surface between two semiinfinite ice sheets, as shown in Fig.1. Each of the ice sheets is assumed to have uniform density, thickness and draught, or $\rho_{1}, h_{1}$ and $d_{1}$ for the left ice sheet and $\rho_{2}, h_{2}$ and $d_{2}$ for the right one, respectively. The density of water is defined as $\rho$. Flexural-gravity waves normal 
to the ice edge propagate beneath one ice sheet from the left infinity to the right. The body on the water surface is a rectangular box with width $a$ and draught $b$, respectively. The horizontal distances on the left and right hand sides between the edges of the ice sheets and the sides of the floating body are $l_{1}$ and $l_{2}$, respectively.

A Cartesian coordinate system $\mathrm{O}-x y z$ is defined where the origin is on the mean free surface and the middle of the body. The $x$-axis is along the direction of the incoming wave while the $z$-axis points upward and $y$-axis into the sheet. The water depth $H$ is assumed to be constant.

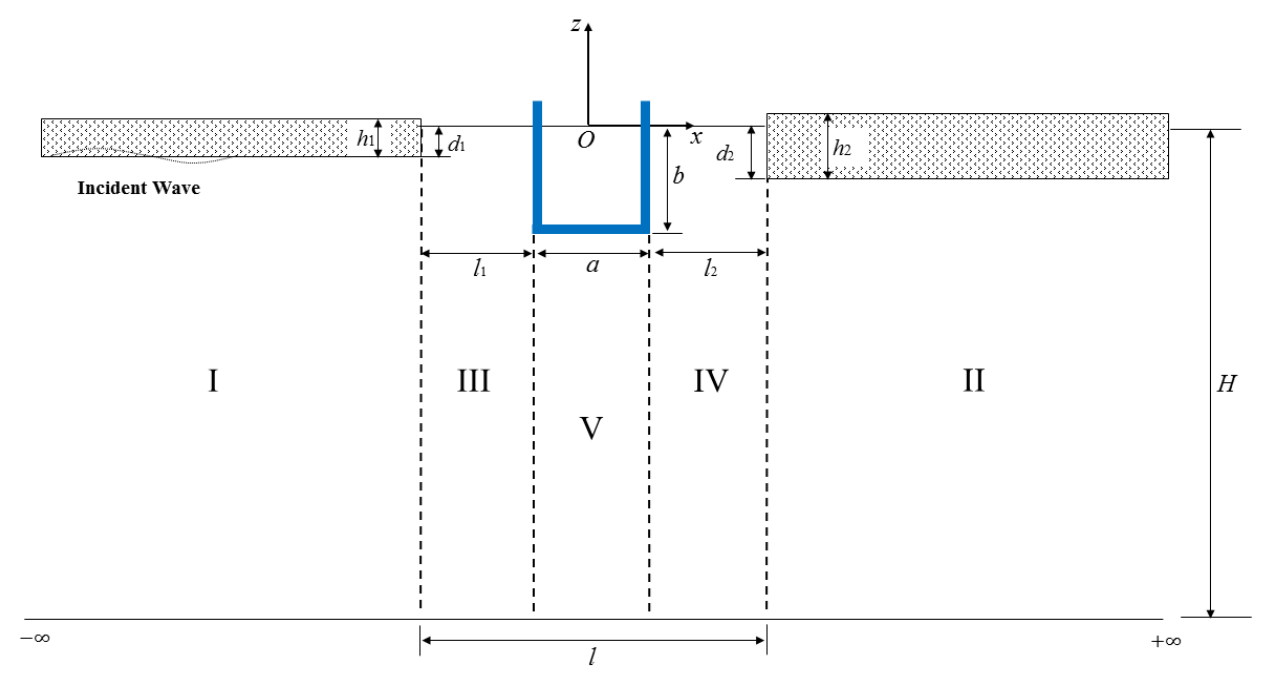

FIG. 1. Geometry and the Cartesian coordinate system of the problem

Based on the assumption that the fluid is ideal, incompressible and its motion is irrotational, the velocity potential $\Phi(x, z, t)$ can be introduced to describe the flow field. For small wave and body amplitude motions, linearization can be introduced. Once motions become sinusoidal in time, the total potential can be decomposed in the following superposition form: ${ }^{24}$

$$
\Phi(x, z, t)=\operatorname{Re}\left\{\phi_{0}(x, z) \times e^{i \omega t}\right\}+\operatorname{Re}\left\{\sum_{j=1}^{3} i \omega \alpha_{j} \phi_{j}(x, z) \times e^{i \omega t}\right\}
$$

where $\phi_{0}(x, z)$ is due to the incident and diffracted waves, while $\phi_{j}(x, z), j=1,2,3$ are due to sway, heave and roll motion and $\alpha_{j}$ is the corresponding complex motion amplitude of the $j^{\text {th }}$ mode. The potential $\phi_{j}(x, z)$ satisfies the following Laplace equation 


$$
\frac{\partial^{2} \phi_{j}}{\partial x^{2}}+\frac{\partial^{2} \phi_{j}}{\partial z^{2}}=0, \quad j=0,1,2,3
$$

in the fluid domain. The linearized boundary condition on the free surface can be written as ${ }^{23}$, 5

$$
g \frac{\partial \phi_{j}}{\partial z}-\omega^{2} \phi_{j}=0,\left(z=0, x_{1}<x<-\frac{a}{2} \text { or } \frac{a}{2}<x<x_{2}\right)
$$

where $x_{1}=-\frac{a}{2}-l_{1}$ and $x_{2}=\frac{a}{2}+l_{2}$, which are the abscissas of the two ice edges, respectively. The boundary conditions on the ice sheet can be written as ${ }^{5}$

$$
\left[L_{i} \frac{\partial^{4}}{\partial x^{4}}-m_{i} \omega^{2}+\rho g\right] \frac{\partial \phi_{j}}{\partial z}-\rho \omega^{2} \phi_{j}=0,\left\{\begin{array}{l}
i=1: z=-d_{1},-\infty<x \leq x_{1} \\
i=2: z=-d_{2}, x_{2} \leq x<+\infty
\end{array}\right.
$$

where $i=1,2$ correspond to the ice sheets in the left and right hand sides, respectively. On the seabed, we have

$$
\frac{\partial \phi_{j}}{\partial z}=0, \quad(z=-H)
$$

In the far field, the radiation condition should be imposed, which requires the disturbed waves to propagate outwards, or

$$
\frac{\partial \phi}{\partial x}= \begin{cases}\kappa_{0} \phi, & x=-\infty \\ -K_{0} \phi, & x=+\infty\end{cases}
$$

where $\kappa_{0}$ and $K_{0}$ are the eigenvalues corresponding to travelling waves in zones I and II, which will be discussed further below. It should be noted that for the diffraction problem, $\phi$ in Eq. (6) refers to the radiation potential $\phi_{j}$ or the diffracted potential ( $\phi_{0}$ without the incident wave). In the above equations, $L_{i}=E h_{i}^{3} /\left[12\left(1-v^{2}\right)\right],(i=1,2)$ are the effective flexural rigidities of the left and right ice sheets, respectively, where $E$ and $v$ are the Young's modulus and Poisson's ratio of the ice. $m_{i}=\rho_{i} h_{i}$ is the mass per unit area of the ice sheet.

On the vertical ice edge surface, the impermeable condition is assumed, which gives

$$
\frac{\partial \phi_{j}}{\partial n}=0,\left(x=x_{i},-d_{i} \leq z \leq 0, i=1,2\right)
$$


On the mean rigid body surface, the boundary condition can be written as

$$
\frac{\partial \phi_{j}}{\partial n}=\left\{\begin{array}{l}
0, j=0 \\
n_{j}, j=1,2,3
\end{array}\right.
$$

where $n_{1}$ and $n_{2}$ are components of the inward normal vector $\boldsymbol{n}$ in $x$ - and $z$-directions, respectively, and $n_{3}=\left(z-z_{0}\right) n_{1}-\left(x-x_{0}\right) n_{2}$ is related to the rotation about the $y$-axis, or the roll motion, with $\left(x_{0}, z_{0}\right)$ being the rotation centre. Specifically, the body surface condition for the rectangular box can be written as

$$
\begin{aligned}
& \frac{\partial \phi_{j}}{\partial x}=\delta_{1 j}+\left(z-z_{0}\right) \delta_{3 j},\left(x=-\frac{a}{2} \text { or } \frac{a}{2},-b \leq z \leq 0, j=1,2,3\right) \\
& \frac{\partial \phi_{j}}{\partial z}=\delta_{2 j}-\left(x-x_{0}\right) \delta_{3 j},\left(-\frac{a}{2} \leq x \leq \frac{a}{2}, z=-b, j=1,2,3\right)
\end{aligned}
$$

where $\delta_{i j}$ is the Kronecker delta function.

It should be noted that the edge condition should also be imposed on the ends of the ice sheets. Similar to an elastic beam, the three common conditions are free edge, simply supported edge and built-in edge, and corresponding mathematical equations for these conditions can be written as follows:

Free edge:

$$
\frac{\partial}{\partial z}\left(\frac{\partial^{2} \phi_{j}}{\partial x^{2}}\right)=\frac{\partial^{2}}{\partial x \partial z}\left(\frac{\partial^{2} \phi_{j}}{\partial x^{2}}\right)=0
$$

Simply supported edge:

$$
\frac{\partial}{\partial z}\left(\frac{\partial^{2} \phi_{j}}{\partial x^{2}}\right)=\frac{\partial \phi_{j}}{\partial z}=0
$$

Built-in edge:

$$
\frac{\partial}{\partial z}\left(\frac{\partial \phi_{j}}{\partial x}\right)=\frac{\partial \phi_{j}}{\partial z}=0
$$

with $j=0,1,2,3$, at $\left(x_{1},-d_{1}\right)$ or $\left(x_{2},-d_{2}\right)$. 


\section{B. Solution procedure}

The fluid domain is divided into five domains shown in Fig.1, namely, $\Omega_{1}\left(-\infty<x \leq x_{1},-H \leq z \leq-d_{1}\right), \Omega_{2}\left(x_{2} \leq x<+\infty,-H \leq z \leq-d_{2}\right), \Omega_{3}\left(x_{1} \leq x \leq-a / 2,-H \leq z \leq 0\right)$ $\Omega_{4}\left(a / 2 \leq x \leq x_{2},-H \leq z \leq 0\right)$ and $\Omega_{5}(-a / 2 \leq x \leq a / 2,-H \leq z \leq-b)$. The method of eigenfunction expansions is adopted to give the expression of velocity potential with unknown coefficients in each domain. Through using the Green's second identity and imposing the continuity conditions of the pressure and velocity on the interface, these coefficients can be found. Specifically, the velocity potential corresponding to the five subdomains are defined as $\phi_{j}^{(i)}$, where the superscript $i=1,2,3 \ldots .5$ refer to the sub-domains. Their expressions, satisfying the Laplace equation and boundary conditions on the horizontal planes, can be written respectively as

$$
\begin{gathered}
\phi_{j}^{(1)}=\delta_{0 j} \phi_{I}+\sum_{m=-2}^{\infty} R_{m}^{(j)} \psi_{m}^{(1)} \\
\phi_{j}^{(2)}=\sum_{m=-2}^{\infty} T_{m}^{(j)} \psi_{m}^{(2)} \\
\phi_{j}^{(3)}=\sum_{m=0}^{\infty} C_{m}^{(j)} \psi_{m}^{(3,1)}+\sum_{m=0}^{\infty} D_{m}^{(j)} \psi_{m}^{(3,2)} \\
\phi_{j}^{(4)}=\sum_{m=0}^{\infty} E_{m}^{(j)} \psi_{m}^{(4,1)}+\sum_{m=0}^{\infty} G_{m}^{(j)} \psi_{m}^{(4,2)} \\
\phi_{j}^{(5)}=\frac{(z+H)^{2}-\left(x+l_{1}+a / 2\right)^{2}}{2(H-b)} \delta_{2 j}-\frac{\left(x-x_{0}\right)(z+H)^{2}-\left(x-x_{0}\right)^{3} / 3}{2(H-b)} \delta_{3 j}+P_{0}^{(j)}\left(x+\frac{a}{2}\right) \\
+S_{0}^{(j)}+\sum_{m=1}^{\infty} P_{m}^{(j)} \psi_{m}^{(5,1)}+\sum_{m=1}^{\infty} S_{m}^{(j)} \psi_{m}^{(5,2)}
\end{gathered}
$$

where

$$
\begin{gathered}
\phi_{I}=\frac{A_{0} g}{i \omega} \times \exp \left[-\kappa_{0}\left(x+\frac{a}{2}+l_{1}\right)\right] \times \frac{\cos \kappa_{0}(H+z)}{\cos \kappa_{0}\left(H-d_{1}\right)} \\
\psi_{m}^{(1)}=\exp \left[\kappa_{m}\left(x+\frac{a}{2}+l_{1}\right)\right] \times \frac{\cos \kappa_{m}(H+z)}{\cos \kappa_{m}\left(H-d_{1}\right)} ; \psi_{m}^{(2)}=\exp \left[K_{m}\left(\frac{a}{2}+l_{2}-x\right)\right] \times \frac{\cos K_{m}(H+z)}{\cos K_{m}\left(H-d_{2}\right)} ; \\
\psi_{m}^{(3,1)}=\exp \left[-k_{m}\left(x+\frac{a}{2}+l_{1}\right)\right] \times \frac{\cos k_{m}(H+z)}{\cos k_{m} H} ; \psi_{m}^{(3,2)}=\exp \left[k_{m}\left(x+\frac{a}{2}\right)\right] \times \frac{\cos k_{m}(H+z)}{\cos k_{m} H} ; \\
\psi_{m}^{(4,1)}=\exp \left[k_{m}\left(\frac{a}{2}-x\right)\right] \times \frac{\cos k_{m}(H+z)}{\cos k_{m} H} ; \psi_{m}^{(4,2)}=\exp \left[k_{m}\left(x-\frac{a}{2}-l_{2}\right)\right] \times \frac{\cos k_{m}(H+z)}{\cos k_{m} H}
\end{gathered}
$$




$$
\psi_{m}^{(5,1)}=\exp \left[\mathscr{\mathscr { T }}_{m}\left(-\frac{a}{2}-x\right)\right] \times \frac{\cos \mathscr{M}_{m}(H+z)}{\cos \mathscr{\mathscr { C }}_{m}(H-b)} ; \psi_{m}^{(5,2)}=\exp \left[\mathscr{K}_{m}\left(x-\frac{a}{2}\right)\right] \times \frac{\cos \mathscr{\mathscr { m }}_{m}(H+z)}{\cos \mathscr{K}_{m}(H-b)}
$$

and $R_{m}^{(j)}, C_{m}^{(j)}, D_{m}^{(j)}, E_{m}^{(j)}, G_{m}^{(j)}, P_{m}^{(j)}, S_{m}^{(j)}$ and $T_{m}^{(j)}$ are unknown coefficients which need to be determined. $A_{0}$ is the amplitude of the incident wave. The constants $k_{m}, \kappa_{m}$ and $K_{m}$ are eigenvalues which are respectively the solutions of the following equations:

$$
\begin{gathered}
-k_{m} \times \tan k_{m} H=\frac{\omega^{2}}{g}, m=0,1,2 \ldots \\
-\kappa_{m} \times \tan \kappa_{m}\left(H-d_{1}\right)=\frac{\rho \omega^{2}}{L_{1} \kappa_{m}^{4}+\rho g-m_{1} \omega^{2}}, m=-2,-1,0,1,2 \ldots \\
-K_{m} \times \tan K_{m}\left(H-d_{2}\right)=\frac{\rho \omega^{2}}{L_{2} K_{m}^{4}+\rho g-m_{2} \omega^{2}}, m=-2,-1,0,1,2 \ldots
\end{gathered}
$$

while $\mathscr{K}_{m}$ can be given as

$$
\mathscr{K}_{m}=\frac{m \pi}{H-b}, m=1,2,3 \ldots
$$

The eigenvalues can be determined from Eqs. (16a)-(16d). In particular, $k_{0}$ is the positive pure imaginary root while $k_{m}(m=1,2,3 \ldots)$ are the positive real roots of Eq. (16a). $\kappa_{0}$ is the positive pure imaginary root; $\kappa_{-2}$ and $\kappa_{-1}$ are the two complex roots with positive real part, while $\kappa_{m}(m=1,2,3)$ are the positive real roots of Eq. (16b). Similarly, $K_{0}$ is the positive pure imaginary root, $K_{-2}$ and $K_{-1}$ are the two complex roots with positive real part, and $K_{m}(m=1,2,3 \ldots)$ are the positive real roots of Eq. (16c).

When functions $\varphi$ and $\psi$ both satisfy Eq. (2), the Green's second identity gives

$$
\oint_{\Gamma}\left(\varphi \frac{\partial \psi}{\partial n}-\psi \frac{\partial \varphi}{\partial n}\right) d s=0
$$

where $\Gamma$ is a closed contour enclosing a fluid domain. For each sub-domain, the above integration along its contour can be applied. When $\varphi$ and $\psi$ satisfy the free surface, bottom and radiation conditions in Eqs. (3), (5) and (6) respectively, the integrations over these surfaces are zero. However, the integration over the ice sheet is non-zero. Using integration by parts on the ice sheet and letting $\varphi=\phi_{j}^{(i)}-\delta_{j 0} \delta_{i 1} \phi_{I}, \psi=\psi_{n}^{(i)}$, Eq. (17) in one of these two domains leads to 


$$
\begin{aligned}
& {\left[\int_{-H}^{-d_{i}}\left(\phi_{j}^{(i)} \frac{\partial \psi_{n}^{(i)}}{\partial x}-\psi_{n}^{(i)} \frac{\partial \phi_{j}^{(i)}}{\partial x}\right) d z\right]_{x=x_{i}}} \\
& +\frac{L_{i}}{\rho \omega^{2}}\left(\frac{\partial^{4} \phi_{j}^{(i)}}{\partial x^{3} \partial z} \frac{\partial \psi_{n}^{(i)}}{\partial z}-\frac{\partial^{3} \phi_{j}^{(i)}}{\partial x^{2} \partial z} \frac{\partial^{2} \psi_{n}^{(i)}}{\partial x \partial z}+\frac{\partial^{2} \phi_{j}^{(i)}}{\partial x \partial z} \frac{\partial^{3} \psi_{n}^{(i)}}{\partial x^{2} \partial z}-\frac{\partial \phi_{j}^{(i)}}{\partial z} \frac{\partial^{4} \psi_{n}^{(i)}}{\partial x^{3} \partial z}\right)_{x=x_{i}, z=-d_{i}}=-\delta_{j 0} \delta_{i 1} \times \zeta, i=1,2 ; j=0,1,2,3
\end{aligned}
$$

where

$$
\zeta=\left\{\begin{array}{l}
\left(\int_{-H}^{-d_{i}}\left(-\phi_{I} \frac{\partial \psi_{n}^{(i)}}{\partial x}+\psi_{n}^{(i)} \frac{\partial \phi_{I}}{\partial x}\right) d z\right)_{x=x_{i}} \\
+\frac{L_{i}}{\rho \omega^{2}}\left(-\frac{\partial^{4} \phi_{I}}{\partial x^{3} \partial z} \frac{\partial \psi_{n}^{(i)}}{\partial z}+\frac{\partial^{3} \phi_{I}}{\partial x^{2} \partial z} \frac{\partial^{2} \psi_{n}^{(i)}}{\partial x \partial z}-\frac{\partial^{2} \phi_{I}}{\partial x \partial z} \frac{\partial^{3} \psi_{n}^{(i)}}{\partial x^{2} \partial z}+\frac{\partial \phi_{I}}{\partial z} \frac{\partial^{4} \psi_{n}^{(i)}}{\partial x^{3} \partial z}\right)_{x=x_{i}, z=-d_{i}}
\end{array}\right\}
$$

$\frac{\partial^{4} \phi_{j}^{(i)}}{\partial x^{3} \partial z}, \frac{\partial^{3} \phi_{j}^{(i)}}{\partial x^{2} \partial z}, \frac{\partial^{2} \phi_{j}^{(i)}}{\partial x \partial z}$ and $\frac{\partial \phi_{j}^{(i)}}{\partial z}$ in Eq. (18) are related to the shear force, bending moment, the slope and the displacement of the ice at its edge. In the present work, without loss of generality, we consider the free edge problem, which suggests that the shear force and bending moment vanish at the edges of the ice sheets. This gives $\frac{\partial^{4} \phi_{j}^{(i)}}{\partial x^{3} \partial z}=\frac{\partial^{3} \phi_{j}^{(i)}}{\partial x^{2} \partial z}=0$ in Eq. (18).

For the sub-domains corresponding to the free surface, $\Omega_{3}$ and $\Omega_{4}$, Eq. (17) gives

$$
\begin{gathered}
{\left[\int_{-H}^{0}\left(-\phi_{j}^{(i)} \frac{\partial \psi_{n}^{(i, l)}}{\partial x}+\psi_{n}^{(i, l)} \frac{\partial \phi_{j}^{(i)}}{\partial x}\right) d z\right]_{x=x_{1}+(i-3) \times\left(a+l_{1}\right)}+\left[\int_{-H}^{0}\left(\phi_{j}^{(i)} \frac{\partial \psi_{n}^{(i, l)}}{\partial x}-\psi_{n}^{(i, l)} \frac{\partial \phi_{j}^{(i)}}{\partial x}\right) d z\right]_{x=-\frac{a}{2}+(i-3) \times\left(a+l_{2}\right)}=0} \\
i=3,4 ; j=0,1,2,3 ; l=1,2
\end{gathered}
$$

For the sub-domain below the floating body, based on the continuity conditions at the interface, we first have

$$
\begin{gathered}
\int_{-H}^{-b} \phi_{j}^{(5)} d z=\int_{-H}^{-b} \phi_{j}^{(3)} d z, \quad x=-\frac{a}{2} \\
\int_{-H}^{-b} \phi_{j}^{(5)} d z=\int_{-H}^{-b} \phi_{j}^{(4)} d z, \quad x=\frac{a}{2}
\end{gathered}
$$


Furthermore, when the Green's identity is applied in $\Omega_{5}$, it gives

$$
\begin{aligned}
& {\left[\int_{-H}^{-b}\left(-\phi_{j}^{(5)} \frac{\partial \psi_{n}^{(5, l)}}{\partial x}+\psi_{n}^{(5, l)} \frac{\partial \phi_{j}^{(5)}}{\partial x}\right) d z\right]_{x=-\frac{a}{2}}+\left[\int_{-\frac{a}{2}}^{\frac{a}{2}}\left(\phi_{j}^{(5)} \frac{\partial \psi_{n}^{(5, l)}}{\partial z}-\psi_{n}^{(5, l)} \frac{\partial \phi_{j}^{(5)}}{\partial z}\right) d z\right]_{z=-b}} \\
& +\left[\int_{-H}^{-b}\left(\phi_{j}^{(5)} \frac{\partial \psi_{n}^{(5, l)}}{\partial x}-\psi_{n}^{(5, l)} \frac{\partial \phi_{j}^{(5)}}{\partial x}\right) d z\right]_{x=\frac{a}{2}}=0, \quad l=1,2
\end{aligned}
$$

Matching procedure in Eqs. (18), (19) and (22) is conducted based on the continuity conditions of pressure and the normal velocity across the interfaces, which transfers the integral equations into a system of linear equations. In particular, when Eq. (18) is used in $\Omega_{1}$, $\phi_{j}^{(1)}$ at $x=x_{1}$ is replaced by $\phi_{j}^{(3)}$ to ensure the pressure continuity. When it is used in $\Omega_{3}$, $\partial \phi_{j}^{(3)} / \partial x$ at $x=x_{1}$ is replaced by $\partial \phi_{j}^{(1)} / \partial x$ at $x=x_{1}$ and by $\partial \phi_{j}^{(5)} / \partial x$ at $x=-a / 2$ to ensure the continuity of the normal velocity. A similar principle is applied on all other interfaces to ensure continuity.

In practical computation, the infinite series in Eqs. (11) to (15) are truncated and the first $N$ terms are kept. $N$ will be chosen to be sufficiently large and its further increase will not affect the desired accuracy. Based on this, Eqs. (18) to (22) can be transformed to a system of linear equations as

$$
\left[\begin{array}{ccc}
A_{11}^{(j)} & \cdots & A_{1,8 N}^{(j)} \\
\vdots & \ddots & \vdots \\
A_{8 N, 1}^{(j)} & \cdots & A_{8 N, 8 N}^{(j)}
\end{array}\right] \cdot\left\{\begin{array}{c}
R_{m}^{(j)} \\
C_{m}^{(j)} \\
D_{m}^{(j)} \\
P_{m}^{(j)} \\
S_{m}^{(j)} \\
E_{m}^{(j)} \\
G_{m}^{(j)} \\
T_{m}^{(j)}
\end{array}\right\}=\left\{\begin{array}{c}
B_{1}^{(j)} \\
B_{2}^{(j)} \\
\vdots \\
B_{8 N-1}^{(j)} \\
B_{8 N}^{(j)}
\end{array}\right\}
$$

In the equation, the coefficients $A_{i j}, B_{i}$ can be obtained explicitly. 


\section{Wave forces and body motions}

After obtaining the expressions for the velocity potential in each sub-domain, the pressure on the wetted body surface can be calculated through the linearized Bernoulli equation. Then, by integrating the pressure along the wetted body surface, the wave forces can be obtained. These forces can be divided into three parts, namely, wave excitation force obtained from the incident and diffracted potentials, radiation force due to oscillation of the body and restoring force. We assume $\boldsymbol{\alpha}=\left(\alpha_{1}, \alpha_{2}, \alpha_{3}\right)$ as the complex displacement amplitude of the body. Then equations for its motion can be obtained ${ }^{24-25}$

$$
\mathcal{A} \cdot \boldsymbol{\alpha}=\mathcal{B}
$$

The coefficients of matrix $\mathcal{A}$ can be written as

$$
\mathcal{A}_{i j}=-\omega^{2}\left(m_{i j}+\mu_{i j}\right)+i \omega \lambda_{i j}+\mathcal{C}_{i j},(i, j=1,2,3)
$$

where $m_{i j}$ and $\mathcal{C}_{i j}$ are the body mass and hydrostatic restoring coefficients, respectively. In the following calculations, we assume that the rotational centre and the mass centre coincide at the centroid of the submerged part of the body, which is $(0,-b / 2)$. Then we have $m_{11}=m_{22}=m=\rho a b$ as the mass of the body and $m_{33}$, as the moment of inertia of the body. The off-diagonal term $m_{i j}$ are equal to zero. The hydrostatic restoring coefficients $\mathcal{C}_{22}=\rho g a$ and $\mathcal{C}_{33}=\rho g a^{3} / 12$ while the rest of $\mathcal{C}_{i j}$ are equal to zero.

The $\mu_{i j}$ and $\lambda_{i j}$ in Eq. (25) are the added mass and damping coefficients, respectively. They can be obtained from

$$
\mu_{i j}-\frac{i \lambda_{i j}}{\omega}=\rho \int_{S_{b}} \phi_{j} n_{i} d s, \quad(i, j=1,2,3)
$$

The damping coefficient $\lambda_{i j}$ can be also calculated by the far-field method (see Appendix B) which can be used to verify the formulation and solution procedure. The column on the right hand side of Eq. (24) corresponds to the wave excitation force $F_{i}$, which can be obtained from

$$
\mathcal{B}_{i}=F_{i}=-i \omega \rho \int_{S_{b}} \phi_{0} n_{i} d s
$$




\section{RESULTS AND DISCUSSION}

\section{A. Convergence test and comparison}

In this section, we choose a polynya case for a convergence test and comparison with previously published results. The floating body is removed from the present model and the number of sub-regions is therefore reduced from five to three. The case was also considered by Sturova, ${ }^{22}$ whose choice of the parameters have been adopted here, namely: $H=500 m, l=100 m, h_{1}=h_{2}=2 m, b=10 m$, together with

$$
E_{1}=E_{2}=5 \mathrm{Gpa}, \rho_{1}=\rho_{2}=922.5 \mathrm{~kg} \mathrm{~m}^{-3}, \rho=1025 \mathrm{~kg} \mathrm{~m}^{-3}, v=0.3 .
$$

The amplitude reflection and transmission coefficients can be respectively defined as

$$
\mathcal{R}=\frac{\left|R_{0}^{(0)}\right|}{|I|}, \quad \mathcal{T}=\frac{\left|T_{0}^{(0)}\right|}{|I|}
$$

where $I=\frac{A_{0} g}{i \omega}$, while $R_{0}^{(0)}$ and $T_{0}^{(0)}$ are the parameters defined in Eqs. (11) and (12), which are related to the waves at $x=-\infty$ and $x=+\infty$, respectively, in the diffraction problem. The above two coefficients satisfy the energy conservation (see Appendix A).

Figure 2(a) gives reflection coefficients for the ice sheets with free edge conditions. It shows that the results from $\mathrm{N}=200$ and $\mathrm{N}=400$ have no graphically visible difference, which suggests that convergence has been achieved. Chung \& Linton ${ }^{19}$ have shown that reflection can be zero at some certain frequencies and this is confirmed in our results. The figure also shows that the present results are in good agreement with those of Sturova ${ }^{22}$, except that there are very small differences at peaks. To further verify the accuracy of the present method, comparison is also made for the case in the top-left subplot of Figure 3 of Chung \& Linton. ${ }^{19}$ It should be noted that Chung \& Linton ${ }^{19}$ used an approximation in dispersion relationship. Here we follow the same procedure by dropping $m_{1}$ and $m_{2}$ terms in Eq. (16). Figure 2(b) shows the comparison and excellent agreement can be found. 


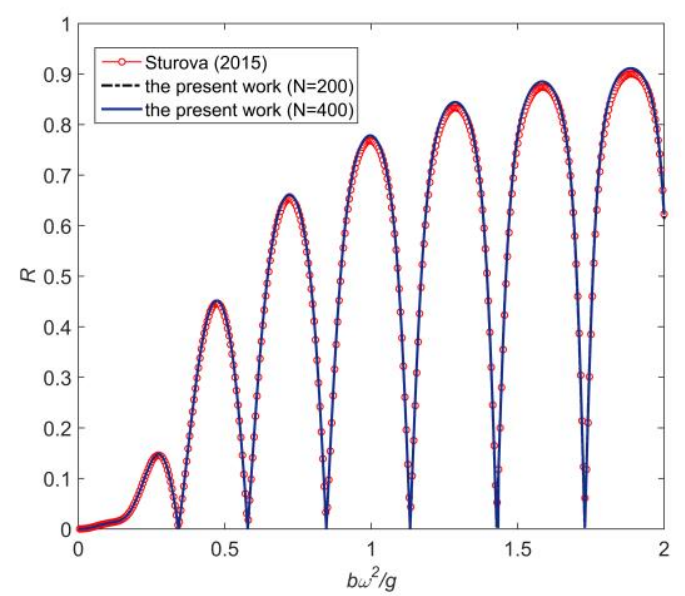

(a)

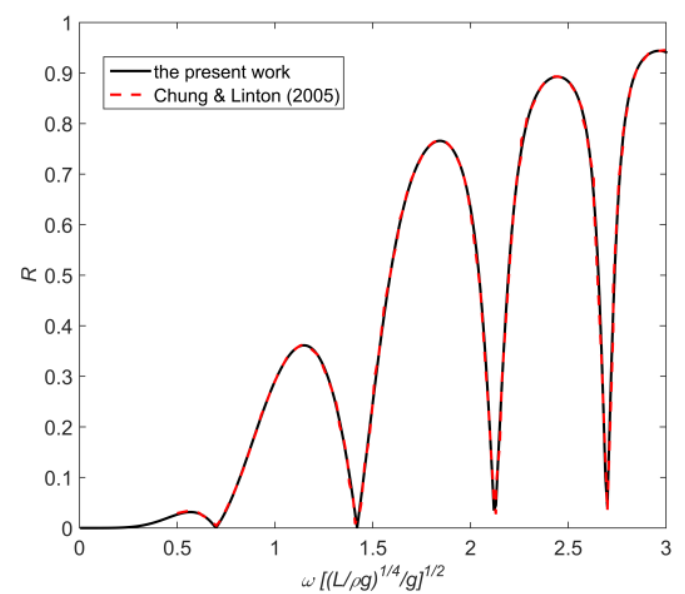

(b)

FIG. 2. Refection coefficient without the floating body in Fig.1: (a) case in Fig.7 of Sturova ${ }^{22}$; (b) case in the top-left subplot of Fig. 3 of Chung \& Linton ${ }^{19}$

\section{B. Hydrodynamic coefficients and excitation force}

We shall use dimensionless variables in the subsequent results and discussions. The width of the box $a$, the density of sea water $\rho$, and the gravitational acceleration $g=9.8 \mathrm{~m} / \mathrm{s}^{2}$ are chosen as basic parameters. All the parameters used below are dimensionlised based on their combinations.

We first consider the wave radiation problem or the body in forced motion. The hydrodynamic coefficients are calculated by using Eq. (26). The added mass and damping coefficients for a floating box confined between two semi-infinite ice sheets with zero and non-zero draughts are shown in Fig. 3, together with the results without the ice sheet. As $\mu_{i j}=\mu_{j i}, \lambda_{i j}=\lambda_{j i}$ (see Appendix B), they are respectively provided as a single curve. The abscissa in the figure is the non-dimensional wavenumber in deep open water, or $\sigma=\omega^{2}$. The convergence of the results with respect to $N$ have been checked and the damping coefficients have also been found to be in excellent agreement with those obtained from the far field equation in the Appendix B. We can see from the figure that the results with zero and nonzero draughts follow the same trend. The effect of the draught is small, if not negligible. This is not entirely unexpected as the mathematical model is based on the assumption that the wavelength is much larger than the thickness of the ice. It is interesting to see that the results with the ice sheets oscillate around those without the ice sheets. We notice 
that at low frequency, or $\omega \rightarrow 0$, the conditions on both the free surface and the ice sheets tend to $\partial \phi_{j} / \partial z=0$, or both the free surface and ice sheets become a rigid lid. Thus the result without ice sheets will be identical to that for the ice sheet of zero draught, which will be slightly different from that with an ice sheet of non-zero draught. As the frequency increases, the results in open water change smoothly. However, the results with ice sheet oscillate continuously. We notice that the wave generated by the body motion in open water will propagate outwards.

When considering the situation with the ice sheet, Eq. (13) shows that while there is a wave moving away from the body (the $D_{0}^{(j)}$ term), there is always a wave moving towards the body (the $C_{0}^{(j)}$ term), which is due to the reflection by the ice sheet on the left hand. Similarly, this occurs in Eq. (14) for zone four. This phenomenon has led to the oscillations in the results. Although the theory is on the basis of large wavelength, we may still consider the mathematical behaviour of the results at high frequency or short wavelength. As $\omega \rightarrow \infty$, the free surface condition becomes $\phi_{j}=0$ on $z=0$. However, the condition on the ice sheet is somewhat different. We notice that the right hand sides of Eqs. (16b) and (16c) become $-\rho / m_{i}$ in such a case and Eq. (4) becomes $\partial \phi_{j} / \partial z+\rho \phi_{j} / m_{i}=0$. This does not correspond to $\phi_{j}=0$. The solutions of Eq. (16b) and (16c) at $m=-2,-1,0$ no longer exist in such a case. In particular, $m=0$ corresponds to the outgoing wave and therefore it does not exist at $\omega=\infty$. In fact, we may notice from Eq. (16b) or Eq. (16c) that the solution at $m=0$ does not exist when the denominator of the right hand side becomes negative. When the denominator is zero, this corresponds to the case of $\phi_{j}=0$ on the ice sheet. However, the wavelength in these cases may be too short for the present model to be used. 


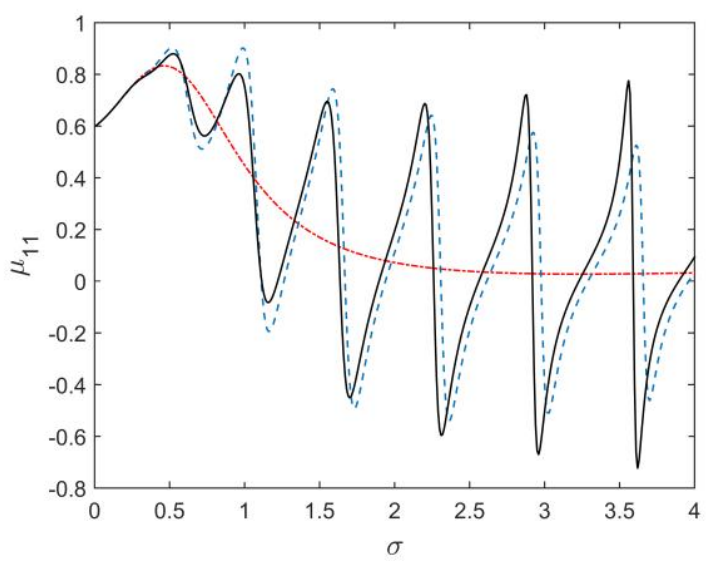

(a)

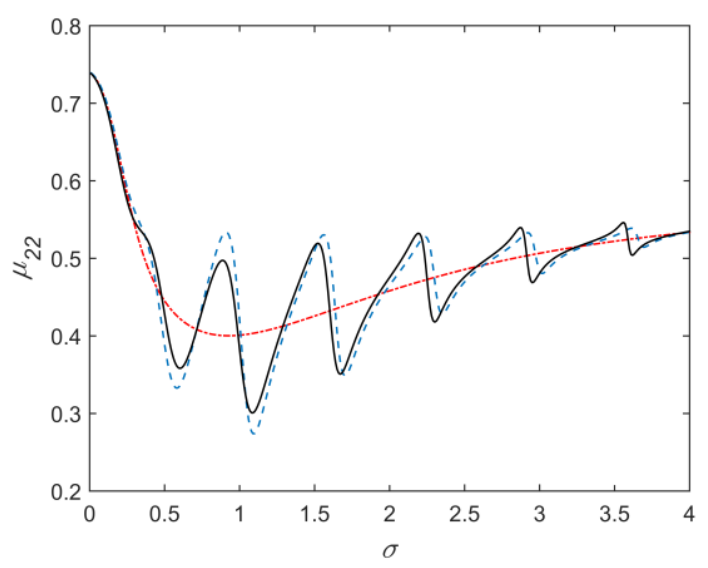

(c)

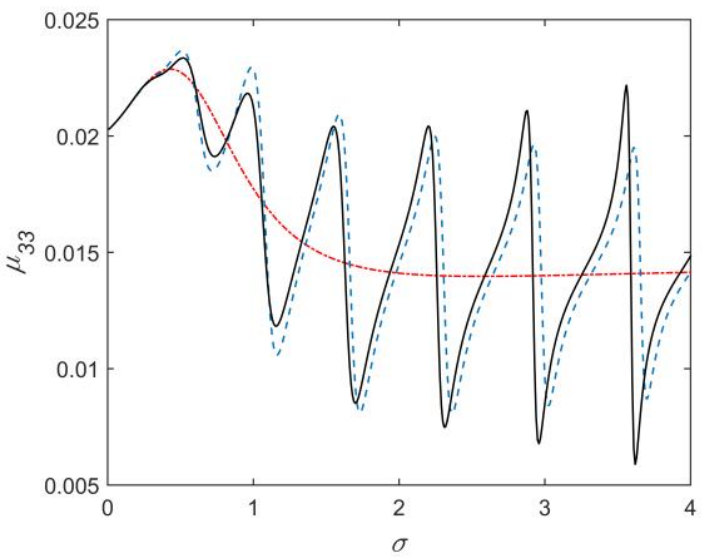

(e)

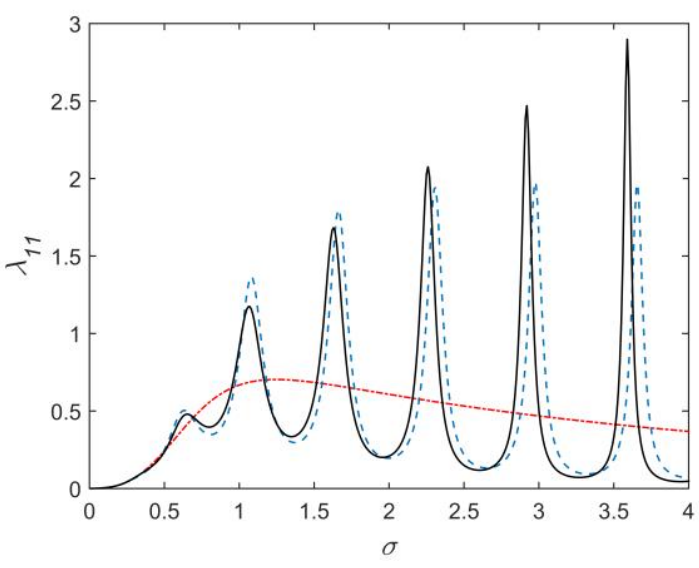

(b)

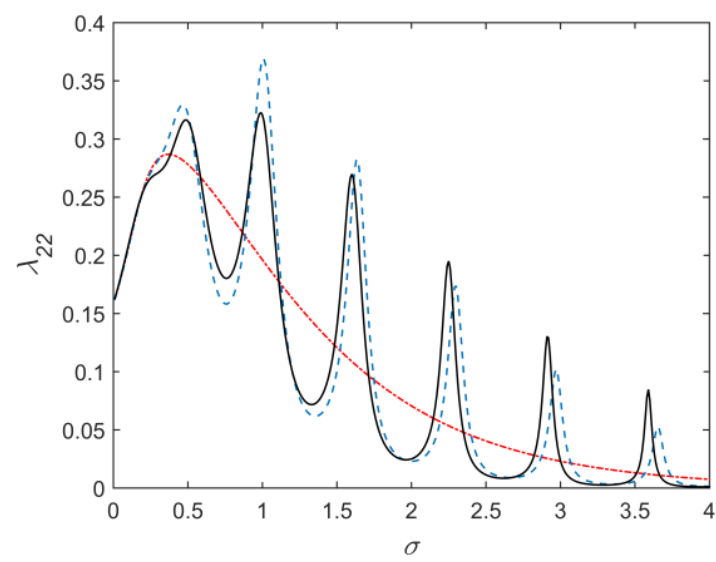

(d)

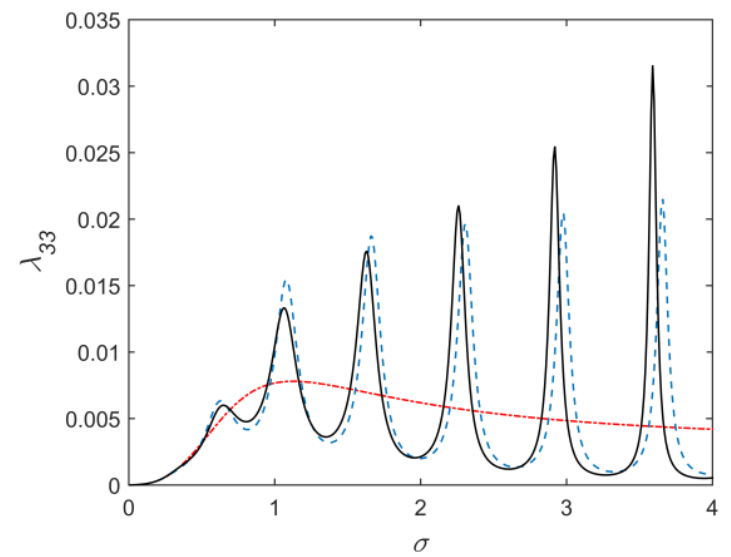

(f) 


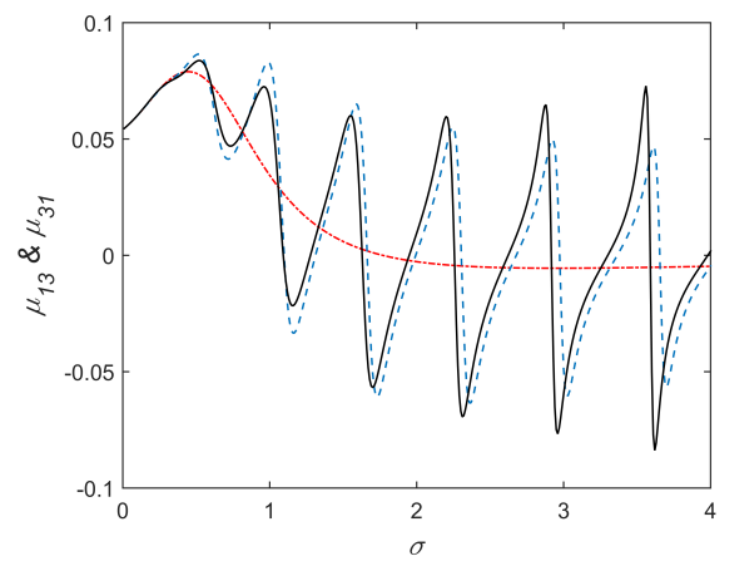

(g)

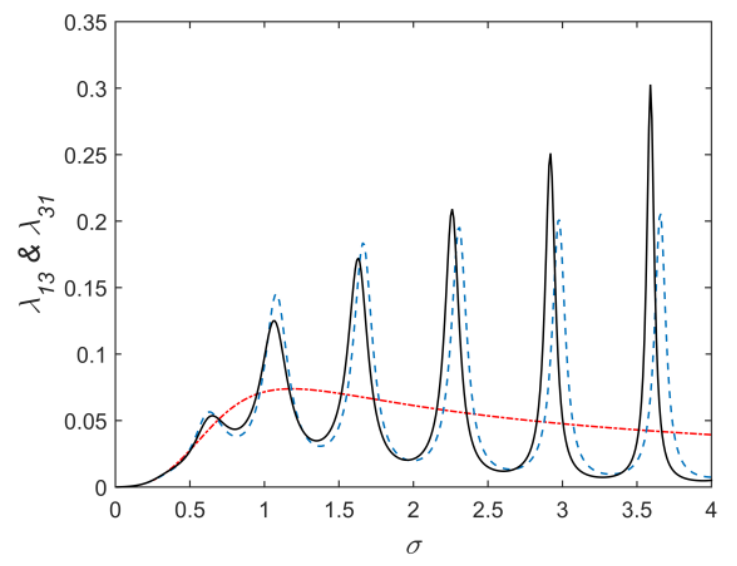

(h)

FIG. 3. The added mass and damping coefficients: dashed line: $d_{l}=d_{2}=0$; solid line: $d_{l}=d_{2}=0.09$; dash-dotted line: without ice sheet ( $a=1, H=10, b=0.5, l_{1}=l_{2}=4.5, m_{1}=m_{2}=0.09, L_{1}=L_{2}=4.5582$ )

We then investigate the effect of the draught of the floating body and the width of the polynya on the hydrodynamic coefficients. Figure 4 shows results of the hydrodynamic coefficients at different floating body draughts, $b$. We can see from the figure that the sway added mass and damping coefficient increase in general with increasing $b$. This is expected as there is less solid surface to push the liquid and the result will obviously zero when $b=0$. For heave, the effect of the disturbance is mainly from the bottom of the body. At smaller $b$, the bottom is closer to the free surface and a larger wave is expected to be created. Therefore, a larger damping coefficient is also expected. For roll, it will be affected by both the sides and bottom of the body. In all these cases, the results are all oscillatory with respect to $\sigma$.

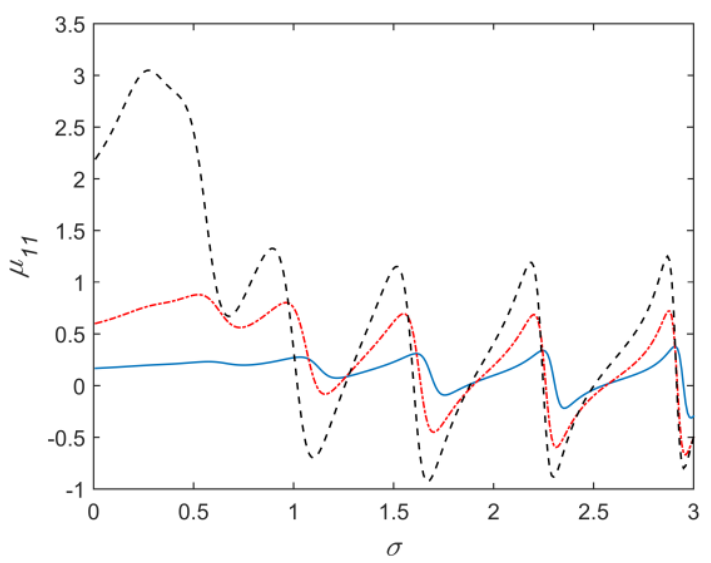

(a)

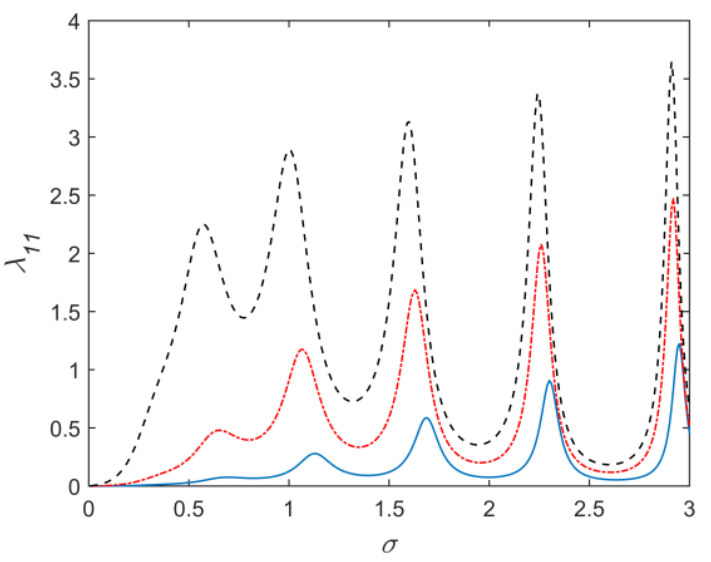

(b) 


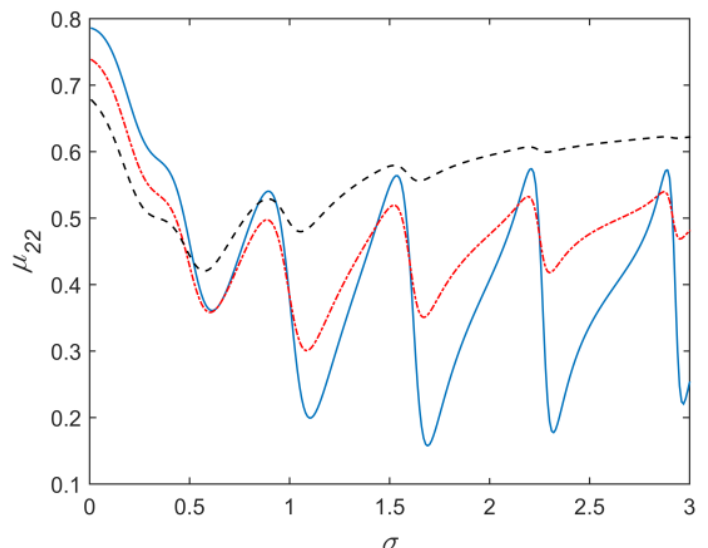

(c)

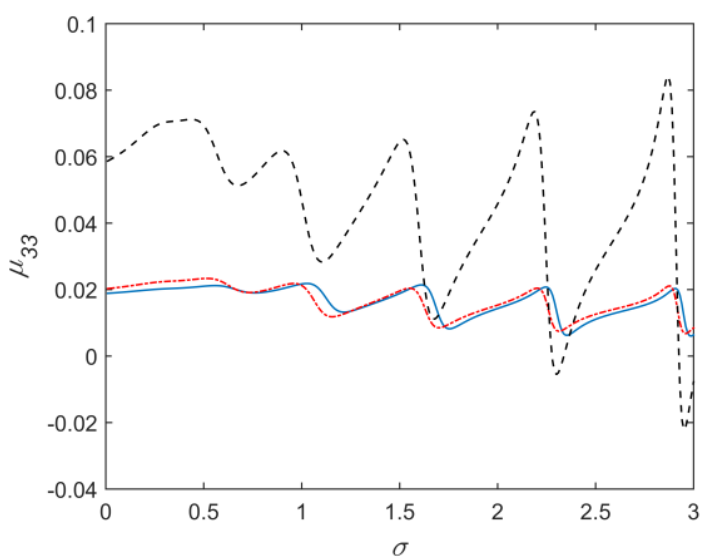

(e)

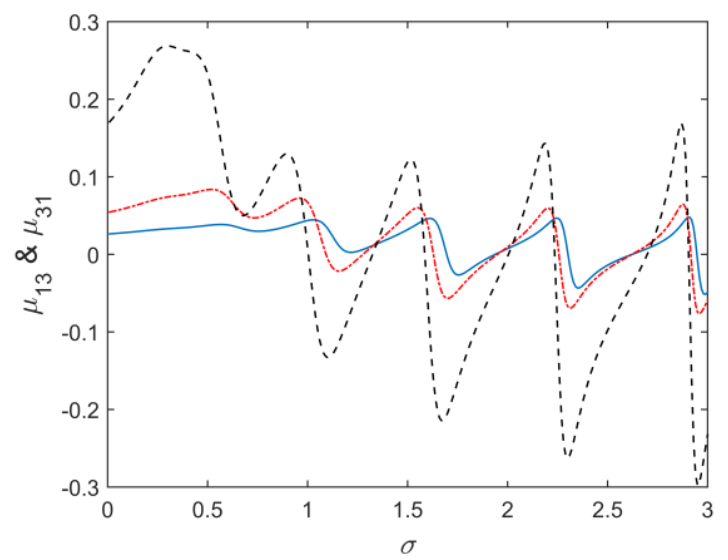

(g)

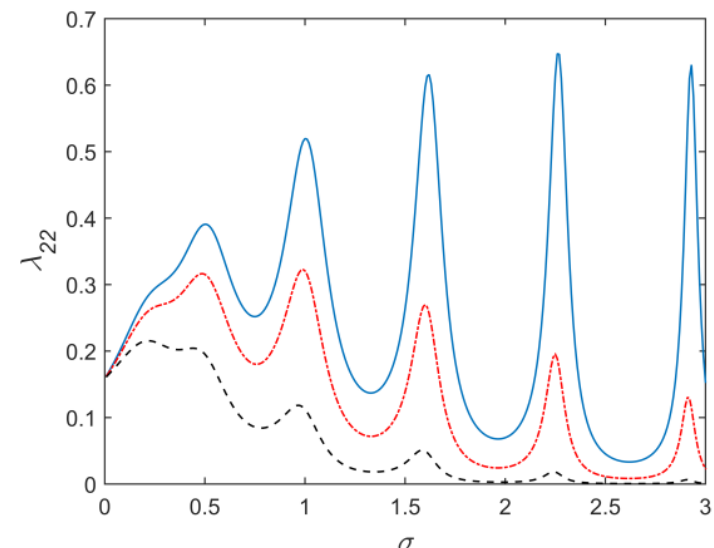

(d)

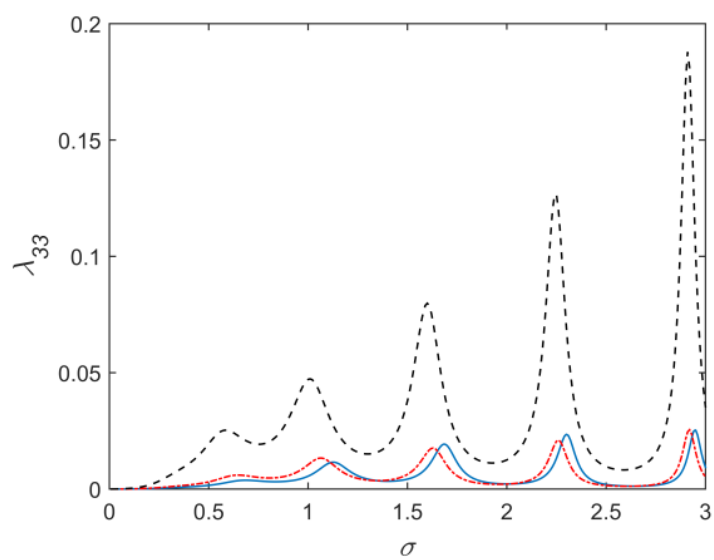

(f)

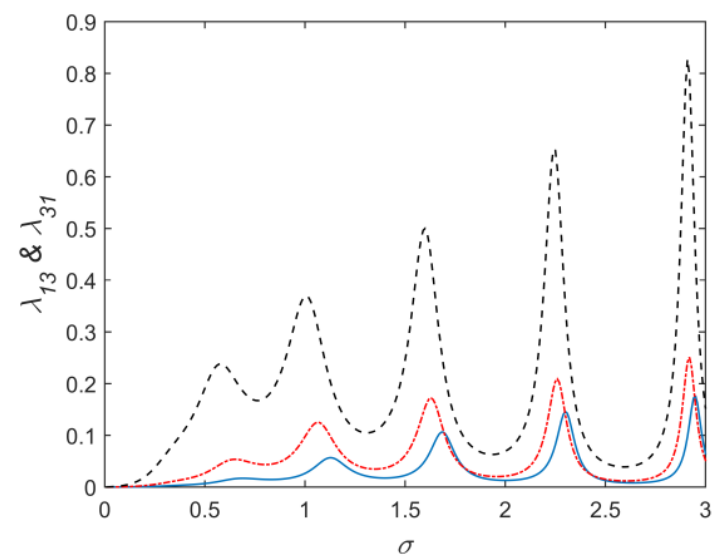

(h)

FIG. 4. Hydrodynamic coefficients $\left(l_{l}=l_{2}=4.5, d_{l}=d_{2}=0.09\right)$ at different draughts: solid lines: $b=0.25$; dash-dotted lines: $b=0.50$; dashed lines: $b=1.00$. (other parameters are the same as those in Fig.3)

Figure 5 shows the effect of polynya width on the hydrodynamic coefficients. It can be seen that the results are less oscillatory with respect to $\sigma$, since the width of the water surface is smaller. This can be linked to the wave elevation in zones III and IV, which will be discussed in Section III D. 


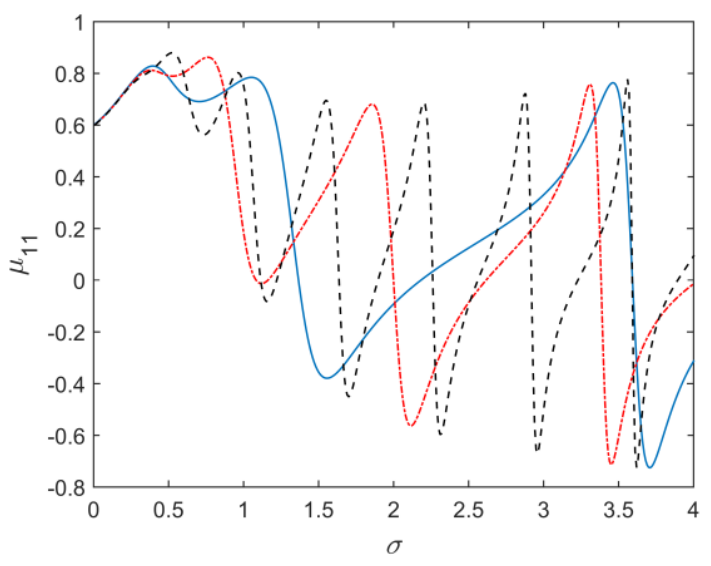

(a)

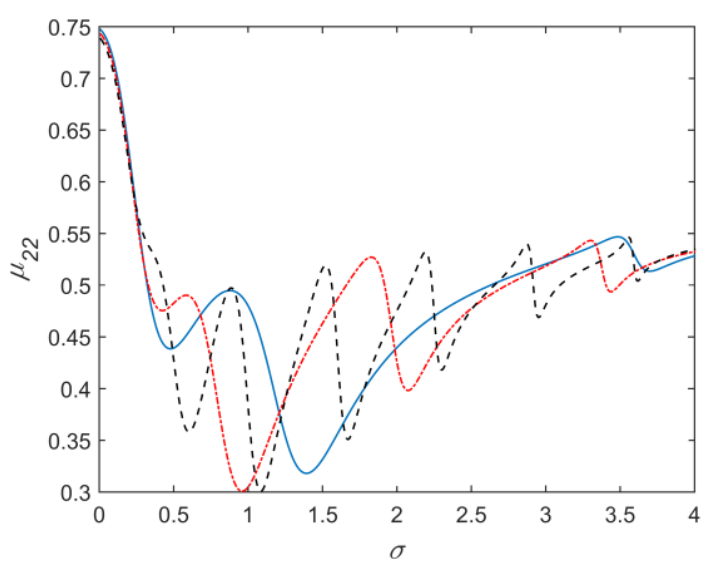

(c)

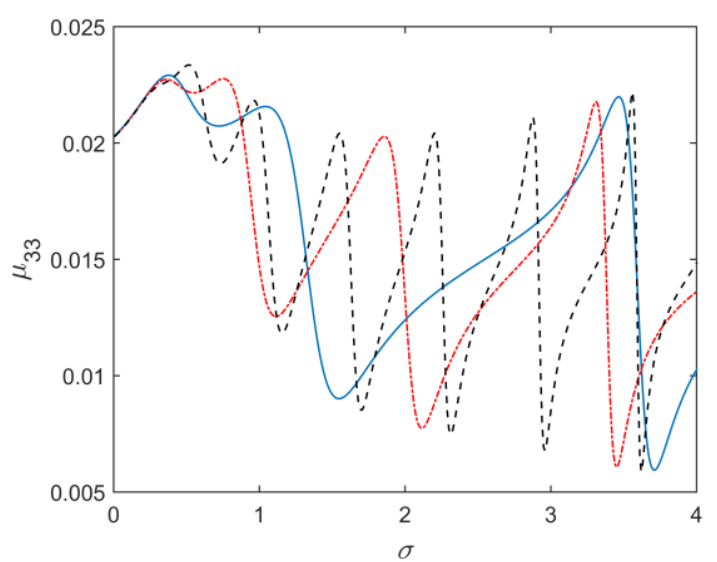

(e)

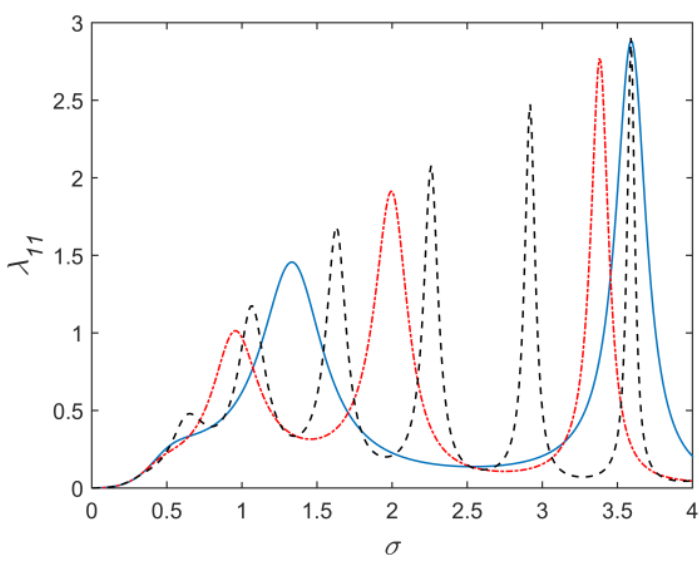

(b)

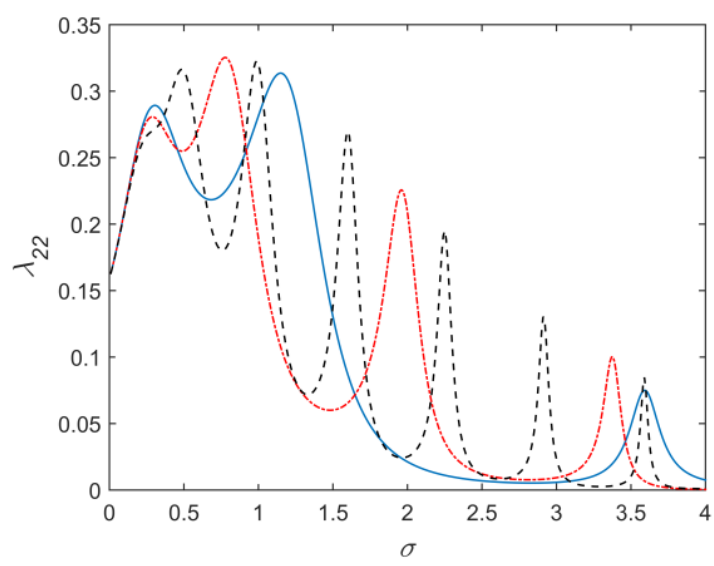

(d)

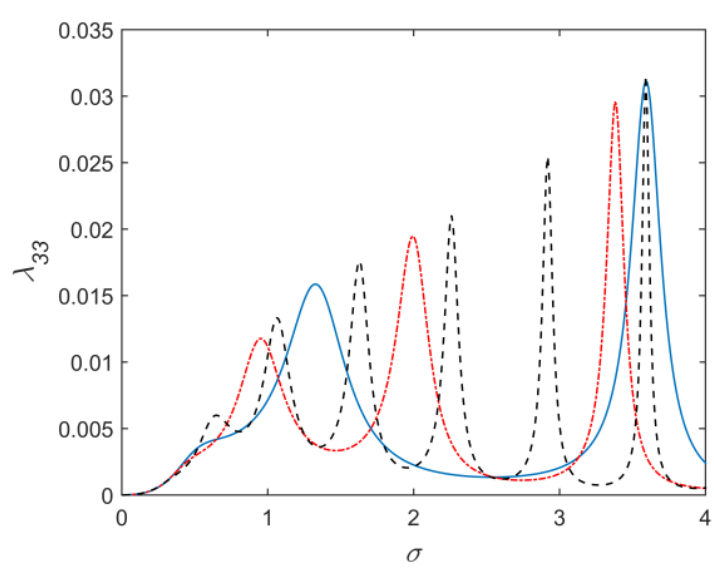

(f) 


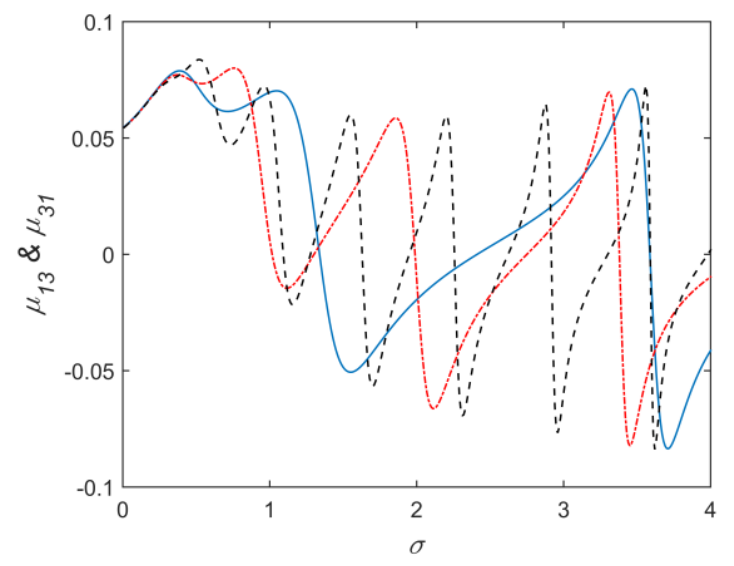

(g)

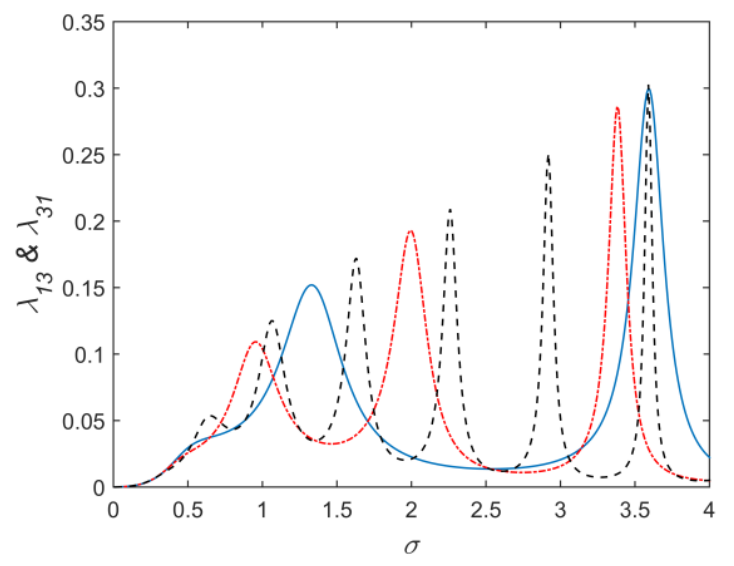

(h)

FIG. 5. Hydrodynamic coefficients $\left(d_{l}=d_{2}=0.09\right)$ at different polynya width: solid lines: $l_{l}=l_{2}=1$; dash-dotted lines: $l_{l}=l_{2}=2$; dashed lines: $l_{1}=l_{2}=4.5$. (Other parameters are the same as those in Fig.3)

We further consider the wave diffraction problem. The excitation forces are calculated based on both Eq. (27) as well as the far field equation (see Appendix B), which are displayed against $\sigma$ in Fig. 6. From the curves, we find that when $\sigma \rightarrow 0$, both $\left|F_{1}\right| / A_{0}$ and $\left|F_{3}\right| / A_{0}$ tend to zero while $\left|F_{2}\right| / A_{0}$ tends to one. These are expected since wavelength in such a case is infinite and much larger than the dimension of the floating body. As the wave number increases, the dynamic effect becomes significant. All the forces oscillate with $\sigma$ and careful inspection shows that $F_{j}$ oscillates in a manner similar to that of $\lambda_{j j}$ in Fig. 5. This is because they are both related to $R_{0}^{(j)}$ in the far field, as shown in the Appendix B.

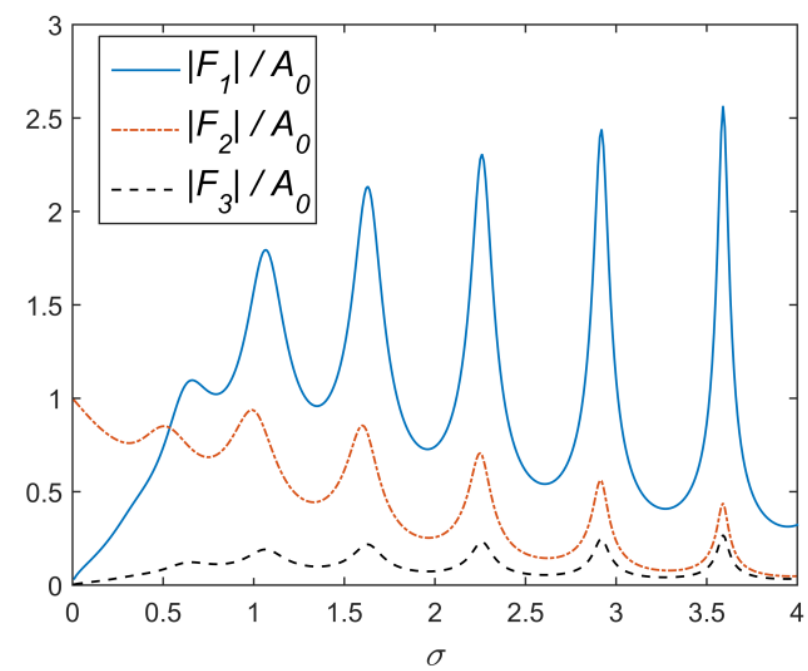

FIG. 6. Excitation forces $\left(d_{l}=d_{2}=0.09\right.$, other parameters are the same as those in Fig. 3$)$ 


\section{Body motions and resonance}

The oscillatory motions of the floating body due to the wave excitation are considered. In Fig. 7, the displacement amplitudes for different modes of motion are displayed for the case of $b=0.5, l_{1}=l_{2}=4.5$ and $m_{33}=0.0521$. The heave motion is symmetric about $x=0$ and is fully decoupled from the coupled sway and roll motions which are anti-symmetric about $x=$ 0 .

A common feature of oscillatory motion is resonance. It happens when the excitation frequency coincides with one of the natural frequencies of the system. The natural frequencies can be found from the un-damped equations for the free motions of the floating body without the excitation force. When the right hand side of Eq. (24) is taken as zero, we have after non-dimensionalisation

$$
\sum_{j=1}^{3} \mathcal{A}_{i j}^{\prime} \alpha_{j}=\sum_{j=1}^{3}\left[-\sigma\left(m_{i j}+\mu_{i j}\right)+\mathcal{C}_{i j}\right] \times \alpha_{j}=0,(i=1,2,3)
$$

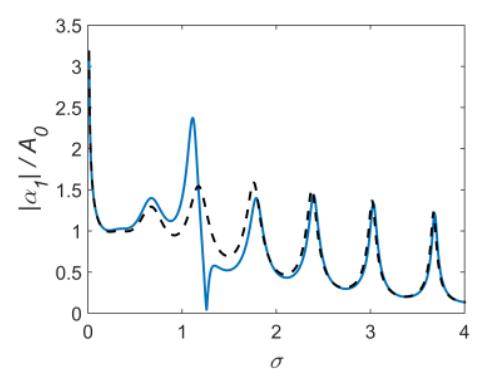

(a). sway

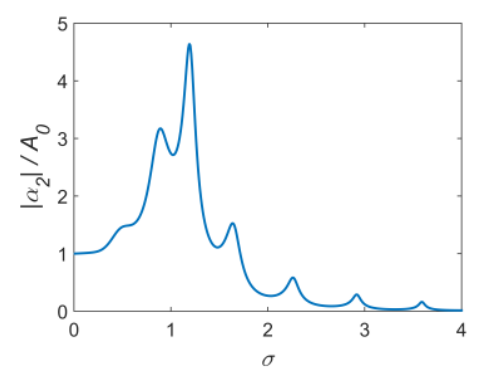

(b). heave

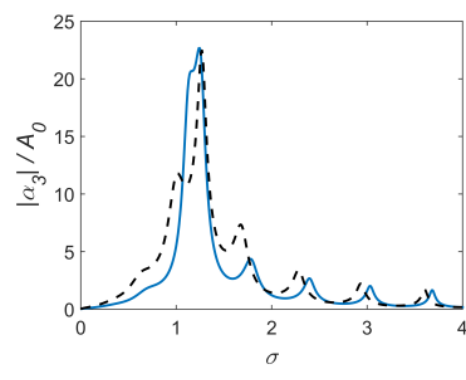

(c). roll

FIG. 7. Motion amplitude with frequency $\left(d_{1}=d_{2}=0.09, m_{33}=0.0521\right.$, other parameters are the same as those in Fig.3): solid lines: coupled motion; dashed lines: uncoupled motion.

where $\sigma=\omega^{2}$ is non-dimensionalised wave number, as defined previously. The natural frequencies of the floating body can be determined from $\left|\mathcal{A}^{\prime}\right|=0$, which, based on Eq. (29), gives two independent equations

$$
-\sigma\left(m_{22}+\mu_{22}\right)+\mathcal{C}_{22}=0
$$

and

$$
\sigma\left[\left(m_{11}+\mu_{11}\right)\left(m_{33}+\mu_{33}\right)-\mu_{13} \mu_{31}\right]-\mathcal{C}_{33}\left(m_{11}+\mu_{11}\right)=0
$$


Eq. (30) in fact corresponds to the uncoupled heave motion while (31) to coupled sway and roll motions. As added mass and damping coefficients in these two equations are frequency dependent, their roots are not able to be obtained explicitly. We may plot two curves with

$$
\sigma_{2}=\frac{\mathcal{C}_{22}}{m_{22}+\mu_{22}}
$$

and

$$
\sigma_{13}=\frac{\mathcal{C}_{33}\left(m_{11}+\mu_{11}\right)}{\left(m_{11}+\mu_{11}\right)\left(m_{33}+\mu_{33}\right)-\mu_{13} \mu_{31}}
$$

as functions of $\sigma$, in Figs.8(a) and 8(b) respectively, in which $m_{11}=m_{22}=0.5, m_{33}=0.0521$, $\mathcal{C}_{22}=1, \mathcal{C}_{33}=1 / 12$ after non-dimensionalisation. The intersections of these curves with the line $f(\sigma)=\sigma$ will be the solutions of Eqs. (30) and (31), respectively. Figure 8(a) shows that there is one natural frequency in heave. When the wave frequency is at the natural frequency, or at the resonance, Fig.7(b) shows a large peak in heave response at around $\sigma \approx 1.19$, which corresponds to the interaction point in Fig.8(a). Multi-intersections can be seen in Fig.8(b). This is principally due to coupling in the two degrees of freedom in oscillation. There exist singular points in the curves of $\sigma_{13}$, which can happen when the denominator in Eq.(32b) or $\mathcal{Z}=\left(m_{11}+\mu_{11}\right)\left(m_{33}+\mu_{33}\right)-\mu_{13} \mu_{31}$ is zero. We notice that $\mu_{13} \mu_{31}=\mu_{13}^{2}$ and $m_{33}+\mu_{33}$ are both positive, while the sign of the term $m+\mu_{11}$ varies with $\sigma$. It can be seen from Fig.3(a) that $\mu_{11}<-0.5$ within some range of frequencies, in which case $\left(m_{11}+\mu_{11}\right)\left(m_{33}+\mu_{33}\right)-\mu_{13} \mu_{31}<0$. When $\mathcal{Z}$ changes sign, $\sigma_{13}$ changes from $+(-) \infty$ to $-(+) \infty$, as reflected in Fig.8(b), leading to multi-intersections with $f(\sigma)$. 


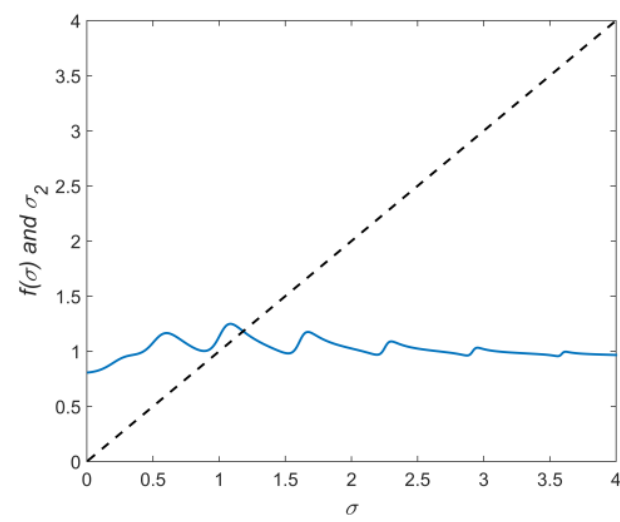

(a)

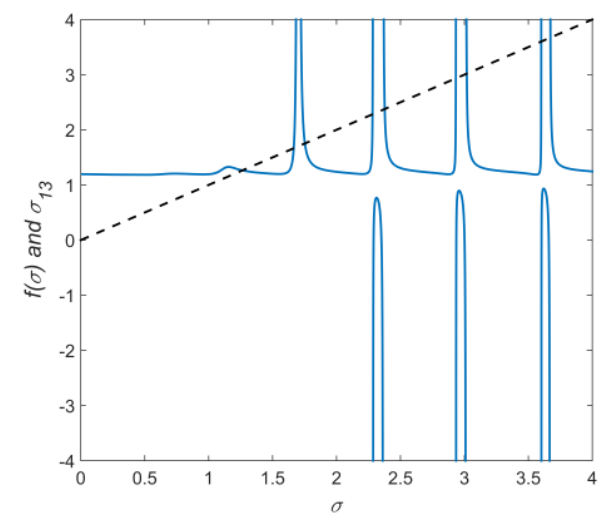

(b)

FIG. 8. Curves based on Eqs. (33a, b): dashed line: $f(\sigma)=\sigma$, the solid line in (a) is for $\sigma_{2}$ while the solid line in (b) is for $\sigma_{13}$.

We notice that the intersection in Fig.8(b) is at around $\sigma=1.26$, at which both sway and roll motions in Figs.7(a) and 7(c) are very big. The motions at other intersections $\sigma=1.75,2.38,3.02,3.67$ are much smaller. It should be pointed out that the natural frequencies obtained from Eqs. (32a) and (32b) are based on the undamped motion, which are usually slightly different from their values with damping. The motion amplitude at resonance also depends on the damping level and the excitation force, both of which are frequency dependent. From Eq. (24), the denominator at resonance is

$$
\mathcal{V}=\omega^{2}\left(\lambda_{13} \lambda_{31}-\lambda_{11} \lambda_{33}\right)-i \omega^{3}\left[\lambda_{11}\left(m_{33}+\mu_{33}-\frac{C_{33}}{\omega^{2}}\right)+\lambda_{33}\left(m_{11}+\mu_{11}\right)-\mu_{13} \lambda_{31}-\mu_{31} \lambda_{13}\right]
$$

We have $|\mathcal{V}|=0.003891,0.03182,0.05522,0.08458,0.1302$ at $\sigma=1.26,1.75,2.38,3.02,3.67$, respectively. This shows at the first intersection of Fig.8(b), the equivalent damping level is very low while at other intersections it is much higher and thus motions are much smaller. Further inspection shows that $|\mathcal{V}|$ depends on the relative value of the various parameters and there is no reason why it can be small or even zero. At resonance, displacement amplitudes of sway and roll motions can become extremely large. To demonstrate this, the rotational inertial $m_{33}$ of the body is adjusted in Fig.7. This changes the natural frequency as well as the value of $|\mathcal{V}|$ in Eq. (33). Figure 9 shows the amplitudes of the sway and roll motions at different $m_{33}$ against $\sigma$. It can be seen that at $m_{33}=0.2$ the motion amplitudes 
can be extremely large. This is principally due to very small $|\mathcal{V}|$, or the equivalent dampling level, although $\lambda_{11}$ and $\lambda_{33}$ are themselves not small.

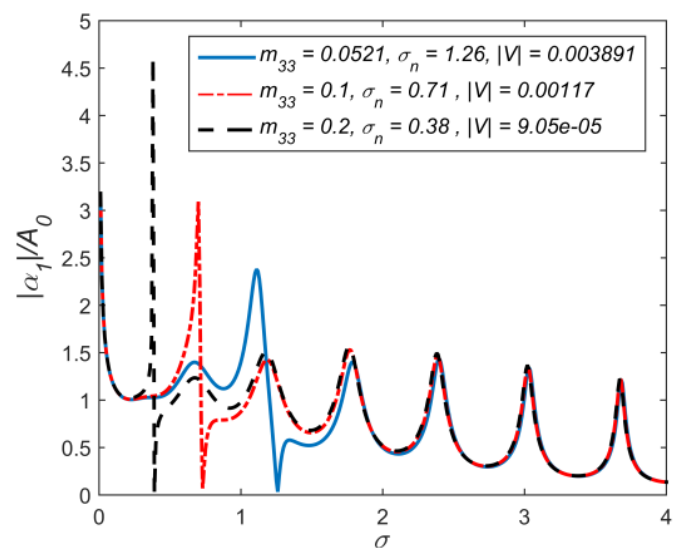

(a)

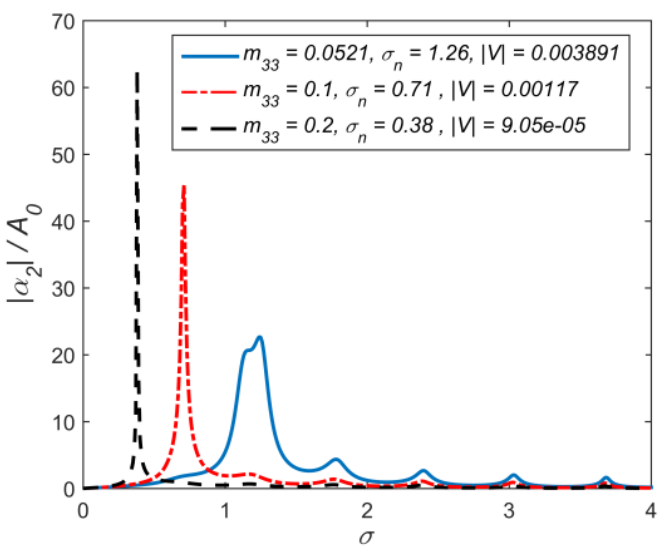

(b)

FIG. 9. Motion amplitude against $\sigma$ at different $m_{33}$. (a): sway; (b): roll. (Other parameters are the same as those in Fig.3)

We also provide results for sway motion when rolling is restrained in Fig.7(a), and roll motion when the sway is restrained in the Fig. 7(c). As the restoring force for the sway motion is zero, its natural frequency is zero. No peak will be expected due to resonance excited by the oscillatory force and the peak in the coupled motion has therefore disappeared. The uncoupled roll motion is on the other hand very similar to the coupled one. Away from resonance there are also other peaks in the motion curves; these are very much related to the oscillatory behaviour of the hydrodynamic coefficients and excitation forces as observed in Section III B.

\section{Surface elevation}

The wave elevation in the polynya is of great interest. Based on the dynamic free surface boundary condition, the wave due to body motion at each mode can be obtained from

$$
\eta_{j}(x)=\frac{\omega^{2}}{g} \alpha_{j} \phi_{j}(x, 0),(j=1,2,3)
$$

Three points on the left side of the body are chosen, as $\eta_{j}(j=1,2,3)$ are either symmetric or anti symmetric about $x=0$. They are respectively at the edge of the ice $x=x_{1}$, middle of the open water $x=-\left(a+l_{1}\right) / 2$ and side of the body $x=-a / 2$. The results obtained from the potential in subdomain III are provided in Fig. 10. From the figure, we can see that the 
elevation amplitude of the three radiated waves at these three typical points have their peak values around the same frequencies. The wave elevation in the figure contains the contribution from all the terms in Eq. (13), which includes those varying exponentially in the $x$ direction. The terms oscillatory with respect to $x$ in the equation are those of $C_{0}^{(j)}$ and $D_{0}^{(j)}$, which have principal contributions to the wave. We notice that the waves corresponding to $C_{0}^{(j)}$ and $D_{0}^{(j)}$ moving towards and moving away from the body respectively. When $\left|C_{0}^{(j)}\right|=\left|D_{0}^{(j)}\right|$, the combination of these two terms would lead to a standing wave on the left hand side of the body. As the radiated wave by each mode of the body motion is either symmetric or anti symmetric, a standing wave will occur simultaneously on the right hand side of the body. Figure 11 shows $\left|R_{0}^{(j)}\right|,\left|C_{0}^{(j)}\right|$ and $\left|D_{0}^{(j)}\right|$ against $\sigma$ respectively for sway, heave and roll motion. We can see that the curves of $\left|C_{0}^{(j)}\right|,\left|D_{0}^{(j)}\right|$ and $\left|R_{0}^{(j)}\right|(j=1,2,3)$ oscillate with $\sigma$ and reach their peak values around the same frequencies. To have some further insights into the links between the local waves due to $C_{0}^{(j)}, D_{0}^{(j)}$, and the radiated wave $R_{0}^{(j)}$, we may assume $l_{1}$ is sufficiently large. In such a case, $D_{0}^{(j)}$ can be approximated as an incoming wave from open water at $x=+\infty, C_{0}^{(j)}$ is the reflection from the semi-infinite ice sheet and $R_{0}^{(j)}$ is the transmitted wave. Then, we can define the reflection and transmission coefficients as those in the case of a semi-infinite ice sheet as follows

$$
\mathcal{R}_{j}=\frac{\left|C_{0}^{(j)}\right|}{\left|D_{0}^{(j)}\right|}, \quad \mathcal{T}_{j}=\frac{\left|R_{0}^{(j)}\right|}{\left|D_{0}^{(j)}\right|}
$$

and they should approximately satisfy the energy conversation ${ }^{6}$

$$
\mathcal{R}_{j}^{2}+\mathcal{D}^{\prime} \times \mathcal{T}_{j}^{2} \approx 1 \quad \text { or } \quad \mathcal{D}^{\prime} \times\left|R_{0}^{(j)}\right|^{2} \approx\left|D_{0}^{(j)}\right|^{2}-\left|C_{0}^{(j)}\right|^{2}
$$

where

$$
\mathcal{D}^{\prime}=\frac{\left\{\frac{2 \kappa_{0}}{\cos ^{2} \kappa_{0}\left(H-d_{1}\right)} \times\left(\frac{\sin 2 \kappa_{0}\left(H-d_{1}\right)}{4 \kappa_{0}}+\frac{H-d_{1}}{2}\right)-\frac{4 L_{1}}{\rho \omega^{2}} \times \kappa_{0}^{5} \tan ^{2}\left[\kappa_{0}\left(H-d_{1}\right)\right]\right\}}{\left\{\frac{2 k_{0}}{\cos ^{2} k_{0} H} \times\left(\frac{\sin 2 k_{0} H}{4 k_{0}}+\frac{H}{2}\right)\right\}}
$$


Eq. (35) establishes a link between the oscillation of $\left|R_{0}^{(j)}\right|$ and those of $\left|C_{0}^{(j)}\right|$ and $\left|D_{0}^{(j)}\right|$ in Fig. 11. Also from the far field equation, the oscillation of the damping coefficients is related to $\left|R_{0}^{(j)}\right|^{2}$, and therefore it is linked to the difference of outgoing wave amplitude squared and the reflected wave amplitude squared. Similarly, as the amplitude of excitation force is related to $\left|R_{0}^{(j)}\right|$ based on the far-field equation, its oscillation can be linked with the wave elevation at infinity. These links can further explain that the location of the peak values in Fig. 11 are approximately the same as those in Fig. 3 for the damping coefficients and Fig. 6 for the excitation forces.

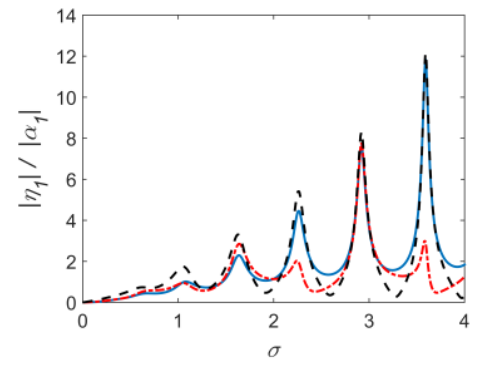

(a)

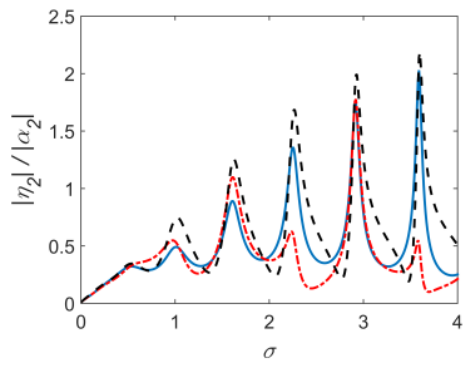

(b)

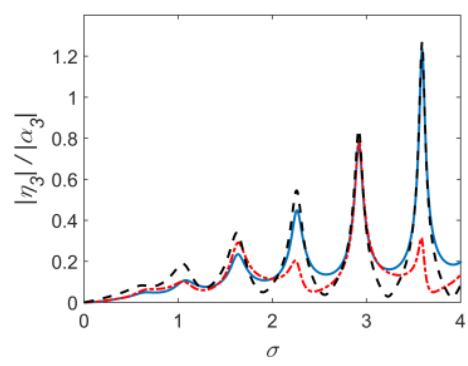

(c)

FIG. 10. The wave elevation amplitude due to body motion (a) sway, (b) heave, (c) roll motion, solid line: $x=x_{1}$; dashdotted line: $x=-\left(a+l_{1}\right) / 2$; dashed line: $x=-a / 2$. (the other parameters are same as those in Fig.3)

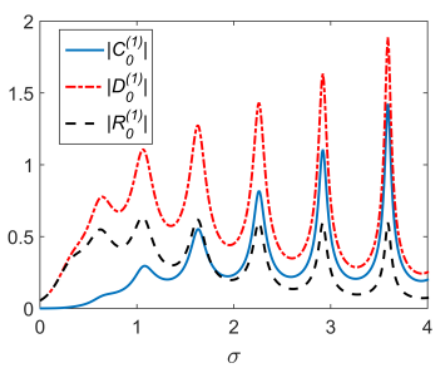

(a)

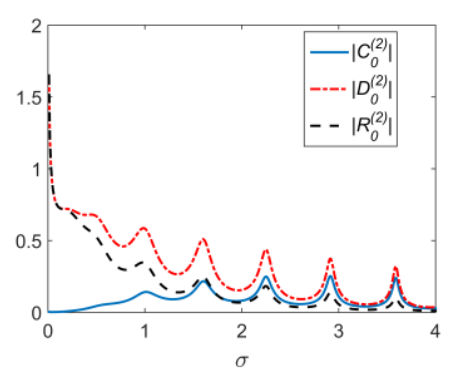

(b)

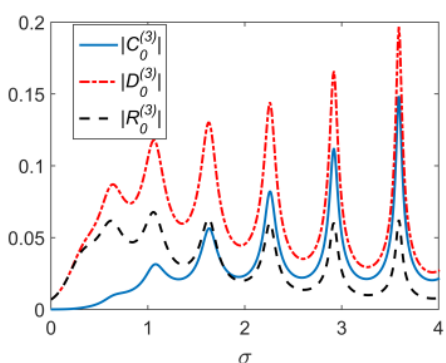

(c)

FIG. 11. $\left|R_{0}^{(j)}\right|,\left|C_{0}^{(j)}\right|$ and $\left|D_{0}^{(j)}\right|$ against $\sigma$. (a) sway, (b) heave, (c) roll motion

$\left(d_{1}=d_{2}=0.09\right.$, other parameters are same as those in Fig.3)

The wave elevation amplitude due to diffracted and incident waves, or $\eta_{0}$, within the subregion of open water can be calculated by replacing $i \omega \alpha_{j} \phi_{j}$ in Eq. (34) with $\phi_{0}$. Since the diffraction problem is not symmetric or anti-symmetric about $x=0$, results are provided in 
Fig. 12 through six points symmetrically arranged on two sides of the body. In Fig. 12(a), it shows that at some wave numbers the wave elevation amplitude is almost zero. As discussed previously, when $\left|C_{0}^{(0)}\right|=\left|D_{0}^{(0)}\right|$, or $\left|E_{0}^{(0)}\right|=\left|G_{0}^{(0)}\right|$, there would be standing waves in zones III or IV, respectively. In Fig. 13, the curves for the ratios of various coefficients to $|I|$ against $\sigma$ are plotted. From Fig.13(a), we can see that the curves for $\left|C_{0}^{(0)}\right| /|I|$ and $\left|D_{0}^{(0)}\right| /|I|$ are almost coincident when $\sigma$ exceeds a certain value, which suggests that standing waves would always occur beyond this point. The amplitude of the standing wave will vary between zero and the summation of those of two single waves, depending on the relative phase of the two waves. The phase changes with $x$ as well as $\sigma$. For a given location, $x=x_{1}$, it is therefore not surprising to have almost zero points at certain wave numbers. Similar behaviour can be observed at $x=-\left(a+l_{1}\right) / 2$, although at different wave numbers, which is due to the fact that both $x$ and $\sigma$ affect the relative phase between the two individual waves. However, near zero points do not occur on the body surface at $x=-a / 2$. This is because the result also contains those terms which vary exponentially with $x$ apart from the terms of $C_{0}^{(0)}$ and $D_{0}^{(0)}$ which vary sinusoidally. It is interesting to see from Fig.12(b) that there are virtually no zero points on the right hand side of the body. This is principally because a standing wave does not occur there as $\left|E_{0}^{(0)}\right|$ and $\left|G_{0}^{(0)}\right|$ do not overlap, as can be seen in Fig.13(b). $\quad\left|R_{0}^{(0)}\right| /|I|$ and $\left|T_{0}^{(0)}\right| /|I|$ are provided in Fig.13(c). They tend to one and zero respectively as wave number increases. When this happens, it means that the incoming wave has completely reflected back towards $x=-\infty$. When the approximation in Eq. (35) is used again in such a case, the wave of $D_{0}^{(0)}$ will not be transmitted into the ice sheet and will be reflected back to water, or $R_{0}^{(0)}=0,\left|C_{0}^{(0)}\right|=\left|D_{0}^{(0)}\right|$. Thus the behaviour in Fig.13(c) is consistent with that in Fig.13(a). 


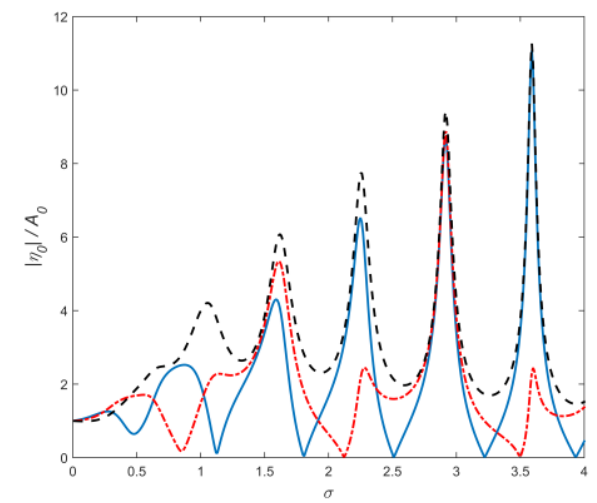

(a)

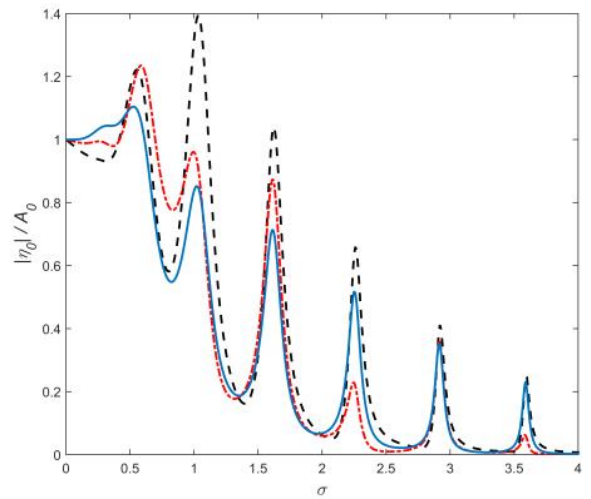

(b)

FIG. 12. The total wave elevation amplitude during wave diffraction and incidence, (a) left hand side of the body: solid line: $x=x_{1}$; dash-dotted line: $x=-\left(a+l_{1}\right) / 2$; dashed line: $x=-a / 2$. (b) right hand side of body: solid line: $x=x_{2} ;$ dashdotted line: $x=\left(a+l_{2}\right) / 2$; dashed line: $x=a / 2 .\left(d_{1}=d_{2}=0.09\right.$, other parameters are same as those in Fig.3)

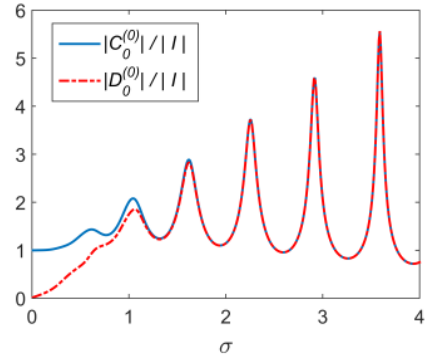

(a)

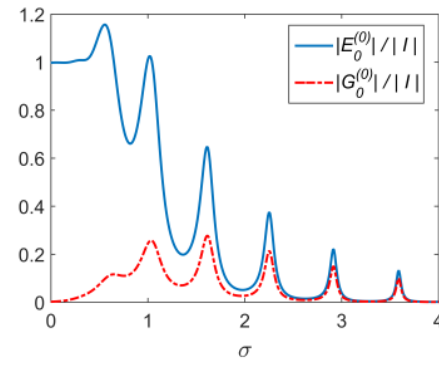

(b)

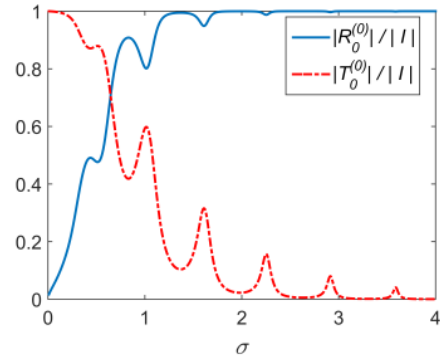

(c)

FIG. 13. Ratios of various coefficients over $|I|$ during wave reflection $\left(d_{1}=d_{2}=0.09\right.$, other parameters are same as those in Fig.3)

\section{CONCLUSION}

The method of matched eigenfunction expansion has been used to investigate the wave excited motion of a body floating on water confined between two semi-infinite ice sheets. The method and solution procedure are verified through a convergence study and comparison with results from existing work through the problem of waves propagating across a polynya. Extensive simulations are then made for a floating rectangular box. Through the obtained results, the following conclusions can be made.

1. Compared with the results in open water, the hydrodynamic coefficients oscillate with respect to the wave frequency. This is principally due to some radiating waves generated by the body motion being reflected back by the ice sheets. 
2. The exciting force on the body has oscillatory behaviour similar to that of hydrodynamic coefficients. In particular, the peaks and troughs of the force curve follow a pattern similar to that of the damping coefficient, as they are both related to the amplitude of the wave generated by the body motion.

3. While there is a single natural frequency in the uncoupled heave motion, there are multi-natural frequencies in the coupled sway and roll motions. The equivalent damping level for the coupled motion is due to the combination of the hydrodynamic coefficients corresponding to sway and roll. It could be very small near the natural frequency and very large motion can be observed in such a case.

4. On the free surface confined by the body and ice, waves propagate in both directions. Standing waves can be observed, especially in the case of wave diffraction. This leads to the wave elevation amplitude at a given location to be zero at certain frequencies.

5. When the gap between the body and ice is large, an approximation based on a wave from infinity can be used. Such an approximation has been found to satisfy the mathematical identity quite accurately.

\section{ACKNOWLEDGEMENTS}

The first author is sponsored by the joint scholarship from University College London and China Scholarships Council, to which he is most grateful. The authors also gratefully acknowledge financial support from the Lloyd's Register Foundation (LRF) through the joint centre involving University College London, Shanghai Jiao Tong University and Harbin Engineering University. The LRF helps to protect life and property by supporting engineering-related education, public engagement and the application of research.

\section{APPENDIX}

\section{A. Relationship between the reflection and transmission coefficients}

When Green's identity is applied for the velocity potential $\phi$ and its complex conjugate over the boundary $\Gamma$ of entire fluid domain in Fig.1, we have

$$
\oint_{\Gamma}\left[\phi \frac{\partial \phi^{*}}{\partial n}-\phi^{*} \frac{\partial \phi}{\partial n}\right] d s=0
$$


By using the boundary conditions, the integration on each boundary will disappear apart from that on the ice sheet and that at infinity. These non-zero integrations can be performed explicitly. Together with the conditions at ice edge, we have

$$
\mathcal{R}^{2}+\mathcal{D} \times \mathcal{T}^{2}=1
$$

where

$$
\mathcal{R}=\frac{\left|R_{0}^{(0)}\right|}{|I|}, \mathcal{T}=\frac{\left|T_{0}^{(0)}\right|}{|I|}
$$

and

$$
\mathcal{D}=\frac{\left\{\frac{2 K_{0}}{\cos ^{2} K_{0}\left(H-d_{2}\right)} \times\left(\frac{\sin 2 K_{0}\left(H-d_{2}\right)}{4 K_{0}}+\frac{H-d_{2}}{2}\right)-\frac{4 L_{2}}{\rho \omega^{2}} K_{0}^{5} \tan ^{2}\left[K_{0}\left(H-d_{2}\right)\right]\right\}}{\left\{\frac{2 \kappa_{0}}{\cos ^{2} \kappa_{0}\left(H-d_{1}\right)} \times\left(\frac{\sin 2 \kappa_{0}\left(H-d_{1}\right)}{4 \kappa_{0}}+\frac{H-d_{1}}{2}\right)-\frac{4 L_{1}}{\rho \omega^{2}} \kappa_{0}^{5} \tan ^{2}\left[\kappa_{0}\left(H-d_{1}\right)\right]\right\}}
$$

This is in fact identical to the case without the floating body. ${ }^{19}$ We notice that for two identical ice plates, $\mathcal{D}=1$.

\section{B. Far-field method for damping coefficient and excitation force}

From Eq. (26), we have

$$
\int_{S_{b}}\left(\phi_{j} \frac{\partial \phi_{k}^{*}}{\partial n}-\phi_{k}^{*} \frac{\partial \phi_{j}}{\partial n}\right) d s=-\frac{2 i}{\rho \omega} \lambda_{k j}
$$

This can be converted to an integration over the rest of the boundary. Applying the boundary conditions, we can obtain

$$
\lambda_{k j}=\frac{\rho \omega}{2 i}\left[\begin{array}{l}
-\frac{L_{1}}{\rho \omega^{2}}\left(4 \kappa_{0}^{5} R_{0}^{(j)} R_{0}^{(k) *} \tan ^{2} \kappa_{0}\left(H-d_{1}\right)\right)-\frac{L_{2}}{\rho \omega^{2}}\left(4 K_{0}^{5} T_{0}^{(j)} T_{0}^{(k) *} \tan ^{2} K_{0}\left(H-d_{2}\right)\right) \\
+2 \kappa_{0} R_{0}^{(j)} R_{0}^{(k) *} \frac{\left(\frac{\sin 2 \kappa_{0}\left(H-d_{1}\right)}{4 \kappa_{0}}+\frac{1}{2}\left(H-d_{1}\right)\right)}{\cos ^{2}\left(\kappa_{0}\left(H-d_{1}\right)\right)}+2 K_{0} T_{0}^{(j)} T_{0}^{(k) *} \frac{\left(\frac{\sin 2 K_{0}\left(H-d_{2}\right)}{4 K_{0}}+\frac{1}{2}\left(H-d_{2}\right)\right)}{\cos ^{2}\left(K_{0}\left(H-d_{2}\right)\right)}
\end{array}\right]
$$

This is similar to that in Sturova. ${ }^{22}$ From Eq. (26), we can also have 


$$
\int_{S_{b}}\left(\phi_{j} n_{i}-\phi_{i} n_{j}\right) d s=\int_{S_{b}}\left(\phi_{j} \frac{\partial \phi_{i}}{\partial n}-\phi_{i} \frac{\partial \phi_{j}}{\partial n}\right) d s=\left(\mu_{i j}-\mu_{j i}\right)-\frac{i}{\omega}\left(\lambda_{i j}-\lambda_{j i}\right)
$$

This can be converted an integration over the rest of the boundary and the result can be found be zero when the boundary conditions are used. Thus

$$
\mu_{i j}=\mu_{j i}, \quad \lambda_{i j}=\lambda_{j i}
$$

If we use Green's second identity for $\phi_{0}$ and $\phi_{j}(j=1,2,3)$ and note that for the diffraction problem $\partial \phi_{0} / \partial n=0$ is satisfied on $S_{b}$, we have

$$
F_{j}=\int_{S_{b}}\left(\phi_{0} \frac{\partial \phi_{j}}{\partial n}-\phi_{j} \frac{\partial \phi_{0}}{\partial n}\right) d s=-\int_{\Gamma-S_{b}}\left(\phi_{0} \frac{\partial \phi_{j}}{\partial n}-\phi_{j} \frac{\partial \phi_{0}}{\partial n}\right) d s
$$

This leads to

$$
F_{j}=-i \omega \rho I R_{0}^{(j)} \times\left[-\frac{4 L_{1}}{\rho \omega^{2}} \kappa_{0}^{5} \tan ^{2} \kappa_{0}\left(H-d_{1}\right)+2 \kappa_{0} \frac{\left(\frac{\sin 2 \kappa_{0}\left(H-d_{1}\right)}{4 \kappa_{0}}+\frac{1}{2}\left(H-d_{1}\right)\right)}{\cos ^{2}\left(\kappa_{0}\left(H-d_{1}\right)\right)}\right]
$$

\section{REFERENCES}

${ }^{1}$ L. C. Smith and S. R. Stephenson. "New Trans-Arctic shipping routes navigable by midcentury." Proceedings of the National Academy of Sciences 110.13, E1191-E1195 (2013).

${ }^{2}$ V. A. Squire, J. P. Dugan, P. Wadhams, P. J. Rottier and A. K. Liu, "Of ocean waves and sea ice." Annual Review of Fluid Mechanics 27.1, 115-168 (1995).

3 V. A. Squire, "Of ocean waves and sea-ice revisited." Cold Regions Science and Technology 49.2 110-133 (2007).

${ }^{4}$ V. A. Squire, "Past, present and impendent hydroelastic challenges in the polar and subpolar seas." Philosophical Transactions of the Royal Society of London A: Mathematical, Physical and Engineering Sciences369.1947, 2813-2831 (2011).

${ }^{5}$ C. Fox, and V. A. Squire. "Reflection and transmission characteristics at the edge of shore fast sea ice." Journal of Geophysical Research: Oceans 95.C7, 11629-11639 (1990). 
${ }^{6}$ C. Fox, and V. A. Squire. "On the oblique reflexion and transmission of ocean waves at shore fast sea ice." Philosophical Transactions of the Royal Society of London A: Mathematical, Physical and Engineering Sciences 347.1682, 185-218 (1994).

${ }^{7}$ T. Sahoo, T. L. Yip, and A. T. Chwang. "Scattering of surface waves by a semi-infinite floating elastic plate." Physics of Fluids (1994-present) 13.11, 3215-3222 (2001).

${ }^{8}$ N. J. Balmforth and R. V. Craster. "Ocean waves and ice sheets." Journal of Fluid Mechanics 395, 89-124 (1999).

${ }^{9}$ L. A. Tkacheva, "Scattering of surface waves by the edge of a floating elastic plate." Journal of Applied Mechanics and Technical Physics 42.4, 638-646 (2001).

${ }^{10}$ L. A. Tkacheva, "The diffraction of surface waves by a floating elastic plate at oblique incidence." Journal of applied mathematics and mechanics 68.3, 425-436 (2004).

$11 \mathrm{H}$. Chung and C. Fox. "Calculation of wave-ice interaction using the Wiener-Hopf technique." New Zealand J. Math 31.1, 1-18 (2002).

${ }^{12}$ D. V. Evans, and T. V. Davies. “Wave-ice interaction.” Rep. 1313. Davidson Lab. Stevens Inst. Technol, Hoboken. NJ (1968)

${ }^{13}$ M. Meylan, and V. A. Squire. "Finite-floe wave reflection and transmission coefficients from a semi-infinite model." Journal of Geophysical Research: Oceans 98.C7,12537-12542 (1993).

${ }^{14}$ M. Meylan, and V. A. Squire. "The response of ice floes to ocean waves." Journal of Geophysical Research-ALL SERIES- 99, 891-891(1994).

${ }^{15}$ T. D. Williams and V. A. Squire. "The effect of submergence on wave scattering across a transition between two floating flexible plates." Wave Motion 45.3, 361-379 (2008).

${ }^{16}$ T. D. Williams and R. Porter. "The effect of submergence on the scattering by the interface between two semi-infinite sheets." Journal of Fluids and Structures 25.5, 777-793 (2009).

17 T. D. Williams and V. A. Squire. "On the estimation of ice thickness from scattering observations." Dynamics of Atmospheres and Oceans 49.2, 215-233 (2010).

18 A. J. Hermans, "Free-surface wave interaction with a thick flexible dock or very large floating platform." Journal of Engineering Mathematics 58.1-4, 77-90 (2007). 
${ }^{19}$ H. Chung and C. M. Linton. "Reflection and transmission of waves across a gap between two semi-infinite elastic plates on water." The Quarterly Journal of Mechanics and Applied Mathematics 58.1, 1-15 (2005).

${ }^{20}$ T. D. Williams and V. A. Squire. "Scattering of flexural-gravity waves at the boundaries between three floating sheets with applications." Journal of Fluid Mechanics 569, 113-140 (2006).

${ }^{21}$ I. V. Sturova, "Wave generation by an oscillating submerged cylinder in the presence of a floating semi-infinite elastic plate." Fluid Dynamics 49.4, 504-514 (2014).

${ }^{22}$ I. V. Sturova, "Radiation of waves by a cylinder submerged in water with ice floe or polynya." Journal of Fluid Mechanics 784, 373-395 (2015).

${ }^{23}$ J. V. Wehausen and E.V. Laitone, "Surface waves." In Handbach des Physik. Springer. Berlin Verlag, 446-778 (1960).

${ }^{24}$ J. N. Newman, Marine Hydrodynamics (MIT Press, Cambridge, 1977).

25 C.-C. Mei, M. Stiassnie, and D. K.-P. Yue, Theory and Applications of Ocean Surface Waves: Linear aspects. (World Scientific, Singapore, 2005). 